\title{
Practical Bipartite Tracking for Networked Robotic Systems via Fixed-Time Estimator-Based Control
}

\author{
Peng Su $\left(\mathbb{D}\right.$, Jinqiang Gan $\left(\mathbb{D}\right.$, Teng-Fei Ding $\mathbb{D}$, Chang-Duo Liang $\mathbb{D}^{\circ}$, and Ming-Feng Ge \\ School of Mechanical Engineering and Electronic Information, China University of Geosciences, Wuhan 430074, China \\ Correspondence should be addressed to Jinqiang Gan; ganjq@cug.edu.cn
}

Received 30 July 2020; Revised 26 April 2021; Accepted 16 May 2021; Published 27 May 2021

Academic Editor: Sergey Dashkovskiy

Copyright (c) 2021 Peng Su et al. This is an open access article distributed under the Creative Commons Attribution License, which permits unrestricted use, distribution, and reproduction in any medium, provided the original work is properly cited.

\begin{abstract}
In this paper, the fixed-time practical bipartite tracking problem for the networked robotic systems (NRSs) with parametric uncertainties, input disturbances, and directed signed graphs is investigated. A new fixed-time estimator-based control algorithm for the NRSs is presented to address the abovementioned problem. By applying a sliding surface and the time base generator (TBG) approach, a new stability analysis method is proposed to achieve the fixed-time practical bipartite tracking for the NRSs. We also derive the upper bound of the convergence time for employing the presented control algorithm to solve the practical bipartite tracking problem and further demonstrate that the convergence time is independent of the initial value. Finally, the simulation examples are given to verify the effectiveness of the presented algorithms.
\end{abstract}

\section{Introduction}

Recently, the cooperative control of the networked robotic systems (NRSs) [1-4] has received increasing attention. The concept of the NRS is to denote a team of the controllable autonomous robots aiming to accomplish single or multiple global tasks over local communication. Compared with a single robot, the NRS is capable of achieving more complex missions in a more effective and flexible way. The distributed control algorithms of multiagent systems have been widely used to obtain various collective behaviors, including target tracking [5, 6], formation control [7-11], containment control [12, 13], distributed optimization [14, 15], distribution networks $[16,17]$, tracking performance limitations $[18,19]$, and resilient control $[20]$ to name a few. Besides, many excellent control algorithms are proposed including observer-based adaptive control [21], fault-tolerant control [22], output feedback fuzzy control $[23,24]$, and fuzzyapproximation-based asymptotic control [25] to solve tracking problem for nonlinear systems. Note that all the abovementioned results are focused on networked systems with only cooperation. However, in some cases of real-world applications, the NRS is required to be divided into two subgroups and executes tasks from two opposite directions. This implies that the subgroups have to compete with each other. Therefore, it is of great significance to study the NRSs with both cooperation and competition.

Recently, bipartite consensus [26-28] and bipartite tracking [29-32] of multiagent systems involving both cooperation and competition have received increasing attention. Compared with conventional coordinated tracking behaviors, the bipartite tracking implies that all the robots converge to two opposite states of the leader with the identical state value and different signs. In [29], a class of bipartite tracking and containment control problems with signed digraphs has been addressed. Considering high-order multiagent system, bipartite tracking problems with uncertainties have been solved in [30]. The bipartite tracking problems subject to the time lag over matrix-weighted signed graphs for the NRSs have been studied in [31]. In [32], the bipartite tracking problems with a dynamic leader have been studied in linear multiagent system. It is worth mentioning that the aforementioned research mainly focused on first-, second-, and higher-order dynamics, as well as Lipschitz-type nonlinear systems. There are only a few results on bipartite tracking problems for the NRSs, and the control approaches for solving such problems are still lacked.

On the contrary, the convergence time is an important performance index in the field of distributed control. It thus motivates the development of the finite-time and the fixed- 
time control to improve such convergence performance and, meanwhile, reject input disturbance [33, 34]. Different from the finite-time control, whose convergence time depends on the initial value $[21,35]$, the fixed-time control can force the states to reach the origin in fixed-time regardless of the initial value [36-42]. Due to such advantage, the fixed-time consensus for nonlinear heterogeneous multiagent systems has been studied in [37]. In [38], the fixed-time tracking for high-order multiagent systems has been taken into account. In [39], the fixed-time control has been applied to secondorder nonlinear multiagent systems by integral sliding-mode approach. Especially, a time base generator (TBG) approach has been delivered to drive the states to approach a desired bounded range in fixed time through adjusting the TBG gain [40]. However, to the best knowledge of the authors, the fixed-time practical bipartite tracking problem for the NRSs remains open.

By the abovementioned discussions, this paper aims to provide a general solution to the fixed-time practical bipartite tracking problem for the NRSs with parametric uncertainties, input disturbances, and directed signed graphs. A fixed-time estimator-based control algorithm is designed to solve this problem. The main contributions of this work are threefold:

(i) Different from the control approaches for achieving the bipartite tracking of multiagent system [29-32], in which the system model is described by first-, second-, and higher-order dynamics, as well as Lipschitz-type nonlinear dynamics. The proposed control algorithms address the bipartite tracking problem of the networked robotic system, in which the dynamics of system is modeled by Euler-Lagrange equation, which is more meaningful to describe the actual physical agents.

(ii) Compared with the existing results on collective behavior of the NRS $[3,4]$, in which the converge time is asymptotical, finite time, which is all related to the initial values of the system, the proposed fixed-time estimator-based control algorithm guarantees that the convergence time is fixed time, which is irrespective of the initial states of the system.

(iii) The presented control algorithm can provide a theoretical framework for stabilizing other complex uncertain networked systems in fixed time.

The remaining parts are organized as follows. Section 2 provides the preliminaries and the problem formulations. In Section 3, the fixed-time estimator-based control algorithm and its stability analysis are proposed. The simulation results are presented in Section 4 to test the algorithm. Finally, the conclusions are summed up in Section 5.

Notation: let $\mathscr{R}^{N}$ be the $N \times 1$ real matrix, $\mathscr{R}^{N \times N}$ be the $N \times N$ real matrix, $\operatorname{diag}(\cdot)$ be the diagonal matrix, and $\operatorname{sgn}(\cdot)$ be the sign function. $\operatorname{sig}^{l}(x)$ is equal to $|x|^{l} \operatorname{sgn}(x)$ with $l>1$. $1_{N}=[1,1, \ldots, 1]^{T}$ is the column vector of the $N$ dimension. $I_{\mathrm{N}}$ is the $N$ dimension identity matrix. $(\cdot)_{\max }$ and $(\cdot)_{\min }$ are the maximum and minimum values of the given vector. $\lambda_{\min }(\cdot)$ is the minimum eigenvalues of the given matrix. Besides, $\|\cdot\|$ is the Euclidean norm.

\section{Preliminaries}

2.1. Graph Theory. The communication of the NRS can be modeled as a directed signed graph $\mathscr{G}=\{\mathscr{V}, \mathscr{E}, \mathscr{A}\}$, where $\mathscr{V}=\{1,2, \ldots, N\}$ is the set of vertexes, $\mathscr{E} \subseteq \mathscr{V} \times \mathscr{V}$ is the set of edges, and $\mathscr{A}=\left[a_{i j}\right] \in \mathscr{R}^{N \times N}$ is the adjacency matrix. An edge $e_{i j} \in \mathscr{E}$ (i.e., $a_{i j} \neq 0$ ) implies that the communication information flows directly from the vertex $j$ to the vertex $i$, otherwise $a_{i j}=0 . a_{i j}>0$ implies that the vertex $i$ cooperates with the vertex $j ; a_{i j}<0$ implies that the vertex $i$ competes with the vertex $j$. Furthermore, assume that $\mathscr{G}$ has no selfloops, i.e., $a_{i i}=0$. A directed path is denoted as a series of edges from $(1,2),(3,4), \ldots,(\eta-1, \eta)$ with distinct vertexes and the length $\eta-1$. A cycle of $\mathscr{G}$ is denoted as the starting and ending vertexes of the path being the same, namely, $\eta=1$. Furthermore, if a cycle has even number negative weights, it is termed as positive cycle; otherwise, a cycle is referred to as a negative cycle. A directed signed graph includes a directed spanning tree, and it implies that there is a rooted vertex which has a directed path to any other vertexes. The Laplacian matrix of $\mathscr{G}$ is defined as $L_{s}=\left[l_{i j}\right] \epsilon$ $\mathscr{R}^{N \times N}$ [26], where $l_{i i}=\sum_{j=1, j \neq i}^{N}\left|a_{i j}\right|$ and $l_{i j}=-a_{i j}$, if $i \neq j$. A directed signed graph $\mathscr{G}$ is detail-balanced if there exist positive constants $h_{1}, h_{2}, \ldots, h_{N}$ such that $h_{j} a_{i j}=h_{i} a_{i j}$. A diagonal weighted matrix $B=\operatorname{diag}\left(a_{10}, \ldots, a_{N 0}\right)$ represents the connection weight between the rooted robot and other robots. In detail, $a_{i 0}>0$ implies that the $i$ th robot can directly receive the information from the rooted robot, otherwise $a_{i 0}=0$.

Definition 1 (see [26]). The directed signed graph is said to be structurally balanced if $\mathscr{V}$ can be grouped into two sets $\mathscr{V}^{+}$and $\mathscr{V}^{-}$satisfying $\mathscr{V}=\mathscr{V}^{+} \cup \mathscr{V}^{-}, \mathscr{V}^{+} \cap \mathscr{V}^{-}=\phi$ and $a_{i j} \geq 0, \forall v_{i}, v_{j} \in \mathscr{V}^{\partial}(\partial \epsilon\{+,-\}), \quad a_{i j} \leq 0, \forall v_{i} \in \mathscr{V}^{\partial}, v_{j} \in \mathscr{V}^{\mathfrak{\ell}}, \partial$ $\neq \wp(\partial, \wp \in\{+,-\})$.

Assumption 1. The directed signed graph $\mathscr{G}$ is structurally balanced and detail-balanced. The augmented graph $\mathscr{\mathscr { G }}$ (including the graph $\mathscr{G}$ and the virtual leader) contains a directed spanning tree with the leader as the rooted vertex.

Lemma 1 (see [26]). A directed signed graph is structurally balanced if and only if there is a diagonal matrix $D=\operatorname{diag}\left(d_{1}, d_{2}, \ldots, d_{N}\right)$ such that DAD is positive semidefinite, where $d_{i}=1$ if $v_{i} \in \mathscr{V}^{+}$and $d_{i}=-1$ if $v_{i} \in \mathscr{V}^{-}$.

Lemma 2 (see [44]). Let $M_{s}=L_{s}+B$ and $M_{D}=D M_{s} D$. If Assumption 1 holds, there exists a positive-definite diagonal matrix $H=\operatorname{diag}\left(h_{1}, h_{2}, \ldots, h_{N}\right)$ such that the matrix $M_{D} H$ is positive definite.

2.2. Time Base Generator. The time base generator (TBG) [43] is defined as a function based on time satisfying predetermined restrictions on its initial and final values. Let the TBG gain be presented as follows: 


$$
g(t)=\frac{\dot{\xi}(t)}{1-\xi(t)+\delta}
$$

where $\xi(t)$ is the TBG and $0<\delta \leq 1$. For any given $t_{f}>0$, we can design a proper TBG $\xi(t)$ such that

(1) $\xi(t)$ is at least $C^{2}$ on $(0,+\infty)$

(2) $\xi(t)$ is continuous and nondecreasing from $\xi(0)=0$ to $\xi\left(t_{f}\right)=1$, where $t_{f}<+\infty$ is a scheduled time constant

(3) $\dot{\xi}(0)=\dot{\xi}\left(t_{f}\right)=0$, where the derivative of $\xi(t)$ at $t=0$ is actually its right derivative

(4) $\xi(t)=1$ and $\dot{\xi}(t)=0$ if $t>t_{f}$

Lemma 3 (see [40]). Considering the following differential system,

$$
\dot{x}(t)=-g(t) x(t), \quad x(0)=x_{0},
$$

where TBG gain $g(t)$ is defined in the form of (1) and $x(t)$ denotes the state. Then, there exists a positive constant $t_{f}>0$ with respect to $\xi(t)$ in (1) such that $\lim _{t \rightarrow t_{f}}|x(t)| \leq \delta / 1+\delta x_{0}$ and $|x(t)| \leq \delta / 1+\delta x_{0}$ on $t \in\left[t_{f}, \infty\right)$, where $\delta$ is given in (1).

Remark 1. An example of the TBG is presented as follows [40]:

$$
\xi(t)=\left\{\begin{array}{l}
\frac{10}{t_{f}^{6}} t^{6}-\frac{24}{t_{f}^{5}} t^{5}+\frac{15}{t_{f}^{4}} t^{4}, \quad 0 \leq t \leq t_{f}, \\
1, \quad t>t_{f},
\end{array}\right.
$$

where $t_{f}>0$ is a positive constant and denotes the userdesigned convergence time, which is an important parameter to ensure the convergence of the states before the time $t_{f}$. Then, the main techniques to design the TBG function is to satisfy four properties given in (1). A typical TBG function with $t_{f}=4$ is shown in Figures 1 and 2 to enhance its visualization.

2.3. System Formulation. The dynamics of the $i$ th robot in the NRS is presented below [36]:

$$
M_{i}\left(q_{i}\right) \ddot{q}_{i}+C_{i}\left(q_{i}, \dot{q}_{i}\right) \dot{q}_{i}+g_{i}\left(q_{i}\right)+d_{i}(t)=\tau_{i},
$$

where $t \geq 0, \quad i \in \mathscr{V}, q_{i}, \dot{q}_{i}, \ddot{q}_{i} \in \mathscr{R}^{n}$ are, respectively, the generalized position, velocity, and acceleration, $M_{i}\left(q_{i}\right) \in \mathscr{R}^{n \times n}$ stands for the positive-definite inertia matrix, $C_{1}\left(q_{i}, \dot{q}_{i}\right) \in \mathscr{R}^{n \times n}$ is the centrifugal-Coriolis matrix, $g_{i}\left(q_{i}\right) \in \mathscr{R}^{n}$ represents the gravitational term, $d_{i}(t) \in \mathscr{R}^{n}$ is the input disturbances satisfying $\sup _{t \geq 0}\left\|d_{i}(t)\right\| \leq d_{M}, d_{M}$ is a positive constant, and $\tau_{i} \in \mathscr{R}^{n}$ denotes the control input. as

The parameters of the dynamical model can be described

$$
\begin{aligned}
M_{i}(q) & =M_{i 0}\left(q_{i}\right)+\Delta M_{i}\left(q_{i}\right), \\
C_{i}\left(q_{i}, \dot{q}_{i}\right) & =C_{i 0}\left(q_{i}, \dot{q}_{i}\right)+\Delta C_{i}\left(q_{i}, \dot{q}_{i}\right), \\
g_{i}\left(q_{i}\right) & =g_{i 0}\left(q_{i}\right)+\Delta g_{i}\left(q_{i}\right),
\end{aligned}
$$

where $M_{i 0}\left(q_{i}\right), C_{i 0}\left(q_{i}, \dot{q}_{i}\right)$, and $g_{i 0}\left(q_{i}\right)$ are the desired parts of the dynamic model and $\Delta M_{i}\left(q_{i}\right), \Delta C_{i}\left(q_{i}, \dot{q}_{i}\right)$, and $\Delta g_{i}\left(q_{i}\right)$

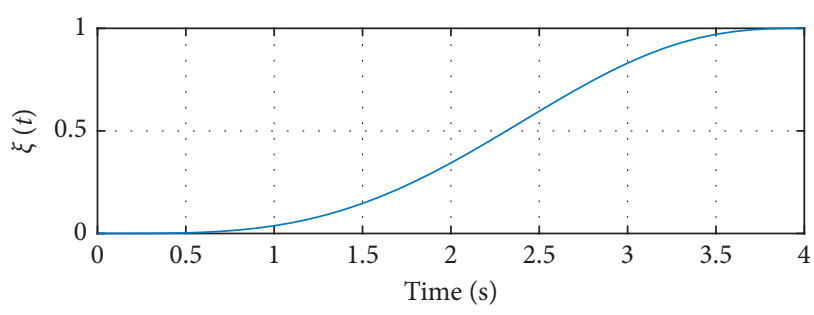

Figure 1: The TBG function.

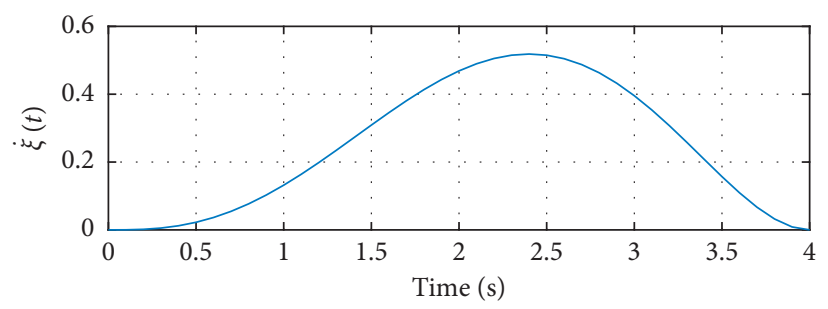

FIgUre 2: The derivative of the TBG function.

are the uncertain term of the dynamic model. Therefore, system (4) can be rewritten as

$$
M_{i 0}\left(q_{i}\right) \ddot{q}_{i}+C_{i 0}\left(q_{i}, \dot{q}_{i}\right) q_{i}+g_{i 0}\left(q_{i}\right)=\tau_{i}+\rho(t),
$$

where $\rho(t)=-\Delta M_{i}\left(q_{i}\right) \ddot{q}_{i}-\Delta C_{i}\left(q_{i}, \dot{q}_{i}\right) \dot{q}_{i}-\Delta g_{i}\left(q_{i}\right)-d_{i}(t)$. Following [36], $\rho(t)$ is upper bounded, namely,

$$
\|\rho(t)\| \leq b_{0}+b_{1}\left\|\dot{q}_{i}\right\|^{2}+b_{2}\left\|\tau_{i}\right\|,
$$

where $b_{0}$ and $b_{1}$ are positive constants and $0<b_{2}<1$. On the contrary, the leader's states $q_{0}, v_{0}$, and $u_{0} \in \mathscr{R}^{n}$ obey that $\dot{q}_{0}=v_{0}$ and $\dot{v}_{0}=u_{0}$.

Assumption 2. The acceleration of the leader is upper bounded, i.e., $\sup _{t \geq 0}\left\|u_{0}(t)\right\| \leq u_{\max }$.

2.4. Problem Formulation. The control objective is to design a proper control input $\tau_{i}$ for achieving the practical bipartite tracking of the NRS in a fixed time. Denote $q_{i}$ and $v_{i}$ as the position and the velocity of the followers. Then, a useful definition and Lemma 4 are listed as follows.

Definition 2. The practical fixed-time bipartite tracking problem is addressed if there exists a positive constant $T$ regardless of the initial value of the states such that $\lim _{t \rightarrow T}\left\|q_{i}(t)-q_{0}(t)\right\| \leq \rho_{1}, \quad \lim _{t \rightarrow T}\left\|v_{i}(t)-v_{0}(t)\right\| \leq \rho_{2}$, $\left\|q_{i}(t)-q_{0}(t)\right\| \leq \rho_{1}$, and $\left\|v_{i}(t)-v_{0}(t)\right\| \leq \rho_{2}$ on $t \in[T, \infty)$, $\forall i \in \mathscr{V}^{+}$, and $\lim _{t \longrightarrow T}\left\|q_{i}(t)+q_{0}(t)\right\| \leq \rho_{1}, \lim _{t \longrightarrow T} \| v_{i}(t)+v_{0}$ $(t)\left\|\leq \rho_{2},\right\| q_{i}(t)+q_{0}(t) \| \leq \rho_{1}$, and $\left\|v_{i}(t)+v_{0}(t)\right\| \leq \rho_{2}$ on $t \in[T, \infty), \forall i \in \mathscr{V}^{-}$, where $\rho_{1}, \rho_{2}>0$ can be sufficiently small by choosing appropriate control parameters.

Lemma 4 (see [33]). For the nonlinear system $\dot{x}=f(x), x(0)=x_{0}, x \in \mathscr{R}^{N}$. Suppose that there exist a positive-definite and continuous function $V(x)$, real numbers $\alpha, \beta>0,0<\gamma_{1}<1$, and $\gamma_{2}>1$ such that $\dot{V}(x)+\alpha V^{\gamma_{1}}(x)+\beta$ $V^{\gamma_{2}}(x) \leq 0$. Then, the origin is the fixed-time stable 
equilibrium point of the considered system. The setting time is given as

$$
T \leq T_{\max }=\frac{1}{\alpha\left(1-\gamma_{1}\right)}+\frac{1}{\beta\left(\gamma_{2}-1\right)}
$$

Remark 2. In missile guidance, hitting the target within a specified time and its predefined neighborhood is a demanding objective. Compare with the traditional control approaches, which can only achieve asymptotic, exponentially, and finite-time stability, because the value of their convergence time depends on the initial condition. Besides, for the complex network, it is very difficult to deal with the switched information between the cyber layer and the physical layer. In the paper, we employ the estimator-based control algorithm to deal with information of each layer. It is a feasible plan for complex cyber-physical system to simplify the proof and make the system stable within fixed time.

Remark 3. The estimator-based control approach is early employed to address the target tracking problem of the networked robotic system in [6]. Based on this idea, the predefined-time formation tracking problem of networked surface vehicles [7], the multiformation tracking problem of networked heterogeneous robotic systems [11], and lagbipartite tracking problem of networked robotic systems [31] have been successfully addressed. It thus believes that the proposed control algorithm can be generalized to other systems and other tracking control problems.

\section{Main Results}

3.1. Fixed-Time Estimator-Based Control Algorithm. In this section, the fixed-time estimator-based control algorithm for achieving practical bipartite tracking of the NRS is proposed. Before constructing the control input, we design the following sliding-mode surface:

$$
s_{i}=\dot{\tilde{q}}_{i}+K_{1 i} \phi\left(\tilde{q}_{i}\right)+K_{2 i} \operatorname{sig}^{l}\left(\widetilde{q}_{i}\right),
$$

where $\tilde{q}_{i}=q_{i}-\widehat{q}_{i}, \dot{\vec{q}}_{i}=\dot{q}_{i}-\dot{\hat{q}}_{i}, \widehat{q}_{i}$ and $\dot{\hat{q}}_{i}$ are the estimated states with respect to $q_{i}$ and $\dot{q}_{i} \in \mathscr{R}^{n}, K_{1 i}, K_{2 i} \in \mathscr{R}^{n \times n}$ are positive-definite diagonal matrixes, $l>1$ is a positive constant, and $\phi\left(\widetilde{q}_{i}\right)=\left(\phi\left(\widetilde{q}_{i 1}\right), \ldots, \phi\left(\widetilde{q}_{i n}\right)\right)$ obeys

$$
\phi\left(\tilde{q}_{i k}\right)= \begin{cases}\operatorname{sig}^{p}\left(\tilde{q}_{i k}\right), & \left|\tilde{q}_{i k}\right| \geq \Delta, \\ p \Delta^{p-1} \tilde{q}_{i k}, & \left|\tilde{q}_{i k}\right|<\Delta, \quad \forall k \in\{1, \ldots, n\},\end{cases}
$$

where $0<\Delta \leq 1$ and $0<p<1$. Besides, for all $k \in\{1, \ldots, n\}$, taking the derivative of $\phi\left(\tilde{q}_{i k}\right)$ with respect to $\tilde{q}_{i k}$ provides that

$$
\nabla \phi\left(\tilde{q}_{i k}\right)=\frac{\partial \phi\left(\tilde{q}_{i k}\right)}{\partial \tilde{q}_{i k}}= \begin{cases}p\left|\tilde{q}_{i k}\right|^{p-1}, & \left|\tilde{q}_{i k}\right| \geq \Delta, \\ p \Delta^{p-1}, & \left|\tilde{q}_{i k}\right|<\Delta .\end{cases}
$$

Let $\nabla \phi\left(\tilde{q}_{i}\right)=\operatorname{col}\left(\nabla \phi\left(\tilde{q}_{i 1}\right), \ldots, \nabla \phi\left(\tilde{q}_{i n}\right)\right)$ and $\zeta\left(\tilde{q}_{i}\right)=$ $\operatorname{col}\left(l\left|\widetilde{q}_{i 1}\right|^{l-1}, \ldots, l\left|\widetilde{q}_{i n}\right|^{l-1}\right)$. The fixed-time estimator-based control algorithm is designed as

$$
\begin{aligned}
& \left\{\begin{array}{l}
\tau_{i}=\tau_{i 0}+\tau_{i 1}+\tau_{i 2}, \\
\tau_{i 0}=M_{i 0}\left(q_{i}\right) \ddot{\vec{q}}_{i}+C_{i 0}\left(q_{i}, \dot{q}_{i}\right) \dot{q}_{i}+g_{i 0}\left(q_{i}\right) \\
\tau_{i 1}=-K_{0 i} \operatorname{sig}^{r}\left(s_{i}\right)-M_{i 0}\left(q_{i}\right)\left(K_{1 i} \operatorname{diag}\left\{\nabla \phi\left(q_{i}\right)\right\}+K_{2 i} \operatorname{diag}\left\{\zeta\left(\tilde{q}_{i}\right)\right\}\right) \dot{\tilde{q}}_{i}, \\
\tau_{i 2}=-\frac{s_{i}}{\left\|s_{i}\right\|} \tau_{\text {aux }} \\
\tau_{\text {aux }}=\frac{1}{1-b_{2}}\left(k+b_{0}+b_{1}\left\|\dot{q}_{i}\right\|^{2}+b_{2}\left\|\tau_{i 0}+\tau_{i 1}\right\|\right)
\end{array}\right. \\
& \left\{\begin{aligned}
\dot{\hat{q}}_{i}(t)= & -\left(\eta_{1}(t)+1\right)\left(\sum_{j=1}^{N}\left|a_{i j}\right|\left(\widehat{q}_{i}(t)-\operatorname{sgn}\left(a_{i j}\right) \widehat{q}_{j}(t)\right)+a_{i 0}\left(\widehat{q}_{i}-d_{i} q_{0}\right)\right) \\
& +\widehat{v}_{i}(t)-c_{1} \operatorname{sgn}\left(\sum_{j=1}^{N}\left|a_{i j}\right|\left(\widehat{q}_{i}(t)-\operatorname{sgn}\left(a_{i j}\right) \hat{q}_{j}(t)\right)+a_{i 0}\left(\widehat{q}_{i}-d_{i} q_{0}\right)\right), \\
\dot{\hat{v}}_{i}(t) & =-\left(\eta_{2}(t)+1\right)\left(\sum_{j=1}^{N}\left|a_{i j}\right|\left(\widehat{v}_{i}(t)-\operatorname{sgn}\left(a_{i j}\right) \hat{v}_{j}(t)+a_{i 0}\left(\widehat{v}_{i}-d_{i} v_{0}\right)\right)\right) \\
& -c_{2} \operatorname{sgn}\left(\sum_{j=0}^{N}\left|a_{i j}\right|\left(\widehat{v}_{i}(t)-\operatorname{sgn}\left(a_{i j}\right) \widehat{v}_{j}(t)\right)+a_{i 0}\left(\widehat{v}_{i}-d_{i} v_{0}\right)\right),
\end{aligned}\right.
\end{aligned}
$$


where (12) represents the local control layer and (13) stands for the estimator layer, $K_{0 i}$ is the positive-definite diagonal matrix, $r>1, k, c_{1}$ and $c_{2}$ are positive constants, $h_{\max }=$ $\max \left\{h_{1}, \ldots, h_{N}\right\}, h_{\min }=\min \left\{h_{1}, \ldots, h_{N}\right\}$, and the TBG gains $\eta_{1}(t)$ and $\eta_{2}(t)$ are defined as

$$
\begin{aligned}
& \eta_{1}(t)=\frac{h_{\max } \dot{\xi}_{1}(t)}{2 \lambda_{\text {min }}\left(\left(M_{D} H\right) \otimes I_{n}\right)\left(1-\xi_{1}(t)+\delta_{1}\right)}, \\
& \eta_{2}(t)=\frac{h_{\max } \dot{\xi}_{2}(t)}{2 \lambda_{\min }\left(\left(M_{D} H\right) \otimes I_{n}\right)\left(1-\xi_{2}(t)+\delta_{2}\right)},
\end{aligned}
$$

$0<\delta_{1}, \delta_{2} \ll 1, \xi_{1}(t)$ and $\xi_{2}(t)$ denoting TBG and $M_{D}$, and $H$ is defined in Lemma 2.

Taking the time derivative of $s_{i}$ and multiplying both sides of the equation by $M_{i 0}(q)$, we have

$$
M_{i 0}\left(q_{i}\right) \dot{s}_{i}=M_{i 0}\left(q_{i}\right) \ddot{\tilde{q}}_{i}+M_{i 0}\left(q_{i}\right)\left(K_{1 i} \operatorname{diag}\left\{\left(\nabla \phi\left(\widetilde{q}_{i}\right)\right\}+K_{2 i} \operatorname{diag}\left\{\zeta\left(\widetilde{q}_{i}\right)\right\}\right) \dot{\tilde{q}}_{i} .\right.
$$

Then, substituting $M_{i 0}\left(q_{i}\right) \ddot{q}_{i}$ from (6) in (15) yields

$$
M_{i 0}\left(q_{i}\right) \dot{s}_{i}=\tau_{i}+\rho(t)-M_{i 0}\left(q_{i}\right) \ddot{\vec{q}}_{i}-C_{0}\left(q_{i}, \dot{q}_{i}\right) \dot{q}_{i}-g_{0}\left(q_{i}\right)+M_{i 0}\left(q_{i}\right)\left(K_{1 i} \operatorname{diag}\left\{\nabla \phi\left(\tilde{q}_{i}\right)\right\}+K_{2 i} \operatorname{diag}\left\{\zeta\left(\tilde{q}_{i}\right)\right\}\right) \dot{\vec{q}}_{i} .
$$

Denote $\widehat{e}_{i}(t)=\widehat{q}_{i}(t)-d_{i} q_{0}$ and $\widehat{\varepsilon}_{i}(t)=\widehat{v}_{i}(t)-d_{i} v_{0}$. Substituting the proposed control input equation $\tau_{i}$ into (16) yields the following cascade closed-loop system:

$$
\left\{\begin{array}{c}
\dot{s}_{i}=M_{i 0}\left(q_{i}\right)^{-1}\left(-\frac{\left.k+b_{0}+b_{1}\left\|\dot{q}_{i}\right\|^{2}+b_{2}\left\|\tau_{i 0}+\tau_{i 1}\right\|_{S_{i}}+\rho(t)-K_{0 i} \operatorname{sig}^{r}\left(s_{i}\right)\right)}{\left(1-b_{2}\right) \| s_{i}||}\right. \\
\dot{\vec{e}}_{i}(t)=-\left(\eta_{1}(t)+1\right)\left(\sum_{j=1}^{N}\left|a_{i j}\right|\left(\widehat{e}_{i}(t)-\operatorname{sgn}\left(a_{i j}\right) \widehat{e}_{j}(t)\right)+a_{i 0} \widehat{e}_{i}\right)+\widehat{\varepsilon}_{i}(t)-c_{1} \operatorname{sgn}\left(\sum_{j=1}^{N}\left|a_{i j}\right|\left(\widehat{e}_{i}(t)-\operatorname{sgn}\left(a_{i j}\right) \widehat{e}_{j}(t)\right)+a_{i 0} \widehat{e}_{i}\right), \\
\dot{\vec{\varepsilon}}_{i}(t)=-\left(\eta_{2}(t)+1\right)\left(\sum_{j=1}^{N}\left|a_{i j}\right|\left(\widehat{\varepsilon}_{i}(t)-\operatorname{sgn}\left(a_{i j}\right) \widehat{\varepsilon}_{j}(t)+a_{i 0} \widehat{\varepsilon}_{i}\right)-c_{2} \operatorname{sgn}\left(\sum_{j=0}^{N}\left|a_{i j}\right|\left(\widehat{\varepsilon}_{i}(t)-\operatorname{sgn}\left(a_{i j}\right) \widehat{\varepsilon}_{j}(t)\right)+a_{i 0} \widehat{\varepsilon}_{i}\right)-d_{i} u_{0}(t) .\right.
\end{array}\right.
$$

Obviously, the fixed-time practical bipartite tracking problem for the NRSs is solved if the closed-loop system (17) is fixed-time stable.

3.2. Stability Analysis for the Distributed Estimator Layer. We firstly focus on the convergence analysis of the distributed estimator layer. It is worthy to point out two positive constants $T_{f 1}$ and $T_{f 2}$ can be arbitrarily determined by selecting proper TBGs. Besides, $\xi_{1}(t)$ and $\xi_{2}(t)$ are predefined in (14).

The position error is represented in the form of $\hat{e}(t)=\left[\hat{e}_{1}^{T}(t), \hat{e}_{2}^{T}(t), \ldots, \hat{e}_{N}^{T}(t)\right]^{T}$. The velocity error is in the form of $\widehat{\varepsilon}(t)=\left[\widehat{\varepsilon}_{1}^{T}(t), \widehat{\varepsilon}_{2}^{T}(t), \ldots, \widehat{\varepsilon}_{N}^{T}(t)\right]^{T}$. Let $\bar{e}(t)=\left(D \otimes I_{n}\right)$ $\hat{e}(t), \bar{\varepsilon}(t)=\left(D \otimes I_{n}\right) \widehat{\varepsilon}(t)$. Then, we can construct the following Lyapunov function candidate:

$$
\begin{aligned}
& V_{1}(t)=\frac{1}{2}\left(\left(M_{D} \otimes I_{n}\right) \bar{e}(t)\right)^{T}\left(\left(M_{D} H\right) \otimes I_{n}\right)^{-1}\left(\left(M_{D} \otimes I_{n}\right) \bar{e}(t)\right), \\
& V_{2}(t)=\frac{1}{2}\left(\left(M_{D} \otimes I_{n}\right) \bar{\varepsilon}(t)\right)^{T}\left(\left(M_{D} H\right) \otimes I_{n}\right)^{-1}\left(\left(M_{D} \otimes I_{n}\right) \bar{\varepsilon}(t)\right) .
\end{aligned}
$$

Denote $\left[z_{i j}\right]_{N n \times N n}=\left(M_{D}^{T} H^{-1}\right) \otimes I_{n}$. Let $z_{\min }=\min \left\{\left\{z_{i i}\right.\right.$ $\left.+\sum_{j \neq i} z_{i j}: z_{i i}+\sum_{j \neq i} z_{i j} \neq 0, \forall i, j \in N n\right\}, \min \left\{\left|z_{i j}\right|: z_{i j} \neq 0, i \neq\right.$ $j, \forall i, j \in N n\}\}\}$.

Theorem 1. Suppose that Assumptions 1-2 hold. If $c_{1} \geq h_{\max } / h_{\min } \sqrt{2 \delta_{2} / z_{\min }\left(1+\delta_{2}\right) V_{2}(0)}$ and $c_{2} \geq N u_{\max } h_{\max }$ $/ h_{\text {min }}$, then estimator (13) yields that 


$$
\begin{gathered}
\lim _{t \longrightarrow T_{f_{1}+T_{f_{2}}}}\left\|\widehat{e}_{i}(t)\right\| \leq \frac{1}{N} \sqrt{\frac{2 \delta_{1}}{z_{\min }\left(1+\delta_{1}\right)} V_{1}(0),} \\
\lim _{t \longrightarrow T_{f_{2}}}\left\|\widehat{\varepsilon}_{i}(t)\right\| \leq \frac{1}{N} \sqrt{\frac{2 \delta_{2}}{z_{\min }\left(1+\delta_{2}\right)} V_{2}(0),} \\
\left\|\widehat{e}_{i}(t)\right\| \leq \frac{1}{N} \sqrt{\frac{2 \delta_{1}}{z_{\min }\left(1+\delta_{1}\right)} V_{1}(0)}, \quad \forall t>T_{f_{1}}+T_{f_{2}}, \\
\left\|\widehat{\varepsilon}_{i}(t)\right\| \leq \frac{1}{N} \sqrt{\frac{2 \delta_{2}}{z_{\min }\left(1+\delta_{2}\right)} V_{2}(0),} \quad \forall t>T_{f_{2}},
\end{gathered}
$$

where $\delta_{1}$ and $\delta_{2}$ are presented in (14). It is equivalent to that $\hat{q}_{i}$ and $\widehat{v}_{i}$ converge to an arbitrarily small neighborhood of the leader's state bilaterally within a bounded convergence time
$T_{f_{1}}+T_{f_{2}}$, where $T_{f_{1}}$ and $T_{f_{2}}$ are the user-designed parameters in the TBG gains $\eta_{1}$ and $\eta_{2}$ presented in (14), following the definition of $g(t)$ and $t_{f}$ provided in Section 2.2.

Proof. The proof is processed in two steps. In the first step, according to (17), the compact form of the velocity error is obtained as $\dot{\bar{\varepsilon}}(t)=-\eta_{2}(t)\left(M_{s} \otimes I_{n}\right) \widehat{\mathcal{\varepsilon}} \quad(t)-\left(M_{s} \otimes I_{n}\right) \widehat{\mathcal{\varepsilon}}(t)$ $-c_{2} \operatorname{sgn}\left(\left(M_{s} \otimes I_{n}\right) \widehat{\mathcal{E}}(t)\right)-\left(D \otimes I_{n}\right)\left(1_{N} \otimes u_{0}\right)$. Since $\mathrm{DD}=I_{N}$, one can obtain that

$$
\dot{\bar{\varepsilon}}=-\left(\eta_{2}(t)+1\right)\left(M_{D} \otimes I_{n}\right) \bar{\varepsilon}-c_{2} \operatorname{sgn}\left(\left(M_{D} \otimes I_{n}\right) \bar{\varepsilon}\right)-1_{N} \otimes u_{0} .
$$

Next, for the given Lyapunov function candidate $V_{2}(t)$, where the matrix $H$ is defined in Lemma 2. Differentiating $V_{2}(t)$ along (21) yields

$$
\begin{aligned}
\dot{V}_{2}(t) & =\left(\left(M_{D} \otimes I_{n}\right) \bar{\varepsilon}(t)\right)^{T}\left(\left(M_{D} H\right) \otimes I_{n}\right)^{-1}\left(\left(M_{D} \otimes I_{n}\right) \dot{\bar{\varepsilon}}(t)\right) \\
& \left.=-\left(\eta_{2}(t)+1\right)\left[\left(\left(M_{D} \otimes I_{n}\right) \bar{\varepsilon}\right)\right)^{T}\left(H^{-1} \otimes I_{n}\right)\left(\left(M_{D} \otimes I_{n}\right) \bar{\varepsilon}\right)\right]-\left(\left(M_{D} \otimes I_{n}\right) \bar{\varepsilon}\right)^{T}\left(H^{-1} \otimes I_{n}\right)\left(c_{2} \operatorname{sgn}\left(\left(M_{D} \otimes I_{n}\right) \bar{\varepsilon}\right)-1_{N} \otimes u_{0}\right) \\
& \leq-\left(\eta_{2}(t)+1\right) h_{\max }^{-1}\left\|\left(M_{D} \otimes I_{n}\right) \bar{\varepsilon}\right\|^{2}-c_{2} h_{\max }^{-1}\left\|\left(M_{D} \otimes I_{n}\right) \bar{\varepsilon}\right\|+h_{\max }^{-1}\left\|\left(M_{D} \otimes I_{n}\right) \bar{\varepsilon}\right\|\left\|1_{N} \otimes u_{0}\right\| \\
& \leq-2 \eta_{2}(t) h_{\max }^{-1} \lambda_{\min }\left(\left(M_{D} H\right) \otimes I_{n}\right) V_{2}(t)+\left(N u_{\max } h_{\max }^{-1}-c_{2} h_{\max }^{-1}\right)\left\|\left(M_{D} \otimes I_{n}\right) \bar{\varepsilon}\right\| \leq-\frac{\dot{\xi}_{2}(t)}{1-\xi_{2}(t)+\delta_{2}} V_{2}(t) .
\end{aligned}
$$

For $t \in\left[0, T_{f_{2}}\right]$, by using Lemma $3, V_{2}(t)$ converges to a residual set $\Omega_{2}=\left\{V_{2}(t) \mid V_{2}(t) \leq \delta_{2} / 1+\delta_{2} V_{2}(0)\right\}$ at a prescribed time $T_{f_{2}}$ independent of initial states of robots, so $\lim _{t \rightarrow T_{f_{2}}}\left\|\bar{\varepsilon}_{i}(t)\right\| \leq 1 / N \sqrt{2 \delta_{2} / z_{\min }\left(1+\delta_{2}\right) V_{2}(0)}$, which is equal to $\lim _{t \rightarrow T_{f_{2}}}\left\|\widehat{\varepsilon}_{i}(t)\right\| \leq 1 / N \sqrt{2 \delta_{2} / z_{\min }\left(1+\delta_{2}\right) V_{2}(0)}$. It indicates that the velocity error can converge to a desired level so long as $\delta_{2}$ is chosen properly in protocol. Moreover, $\left\|\widehat{\varepsilon}_{i}(t)\right\| \leq 1 / N \sqrt{2 \delta_{2} / z_{\min }\left(1+\delta_{2}\right) V_{2}(0)}, \forall t>T_{f_{2}}$ and $\lim _{t \rightarrow+}$ $\infty\left\|\widehat{\varepsilon}_{i}(t)\right\|=0$. That is, $\widehat{v}_{i} \longrightarrow v_{0}, \forall i \in \mathscr{V}^{+} ; \widehat{v}_{i} \longrightarrow-v_{0}, \forall$ $i \in \mathscr{V}^{-}$.

In the second step, according to (17), the compact form of the position error is expressed as $\dot{\hat{e}}(t)=-\eta_{1}(t)\left(M_{s} \otimes\right.$ $\left.I_{n}\right) \hat{e}(t)-\left(M_{s} \otimes I_{n}\right) \quad \widehat{e}(t)+\widehat{v}(t)-\left(D \otimes I_{n}\right)\left(1_{N} \otimes v_{0}\right)-c_{1} \operatorname{sgn}$ $\left(\left(M_{s} \otimes I_{n}\right) \widehat{e}\right)$. Then, one may further obtains that

$$
\dot{\bar{e}}(t)=-\left(\eta_{1}(t)+1\right)\left(M_{D} \otimes I_{n}\right) \bar{e}+\left(D \otimes I_{n}\right)\left(\widehat{v}-\left(D \otimes I_{n}\right)\left(1_{N} \otimes v_{0}\right)\right)-c_{1} \operatorname{sgn}\left(\left(M_{D} \otimes I_{n}\right) \bar{e}\right) .
$$

Next, for the given following Lyapunov function candidate $V_{1}(t)$, differentiating $V_{1}(t)$ along (23) yields that

$$
\begin{aligned}
\dot{V}_{1}(t) & =\left(\left(M_{D} \otimes I_{n}\right) \bar{e}(t)\right)^{T}\left(\left(M_{D} H\right) \otimes I_{n}\right)^{-1}\left(\left(M_{D} \otimes I_{n}\right) \dot{\bar{e}}(t)\right) \\
& =-\left(\eta_{1}(t)+1\right)\left[\left(\left(M_{D} \otimes I_{n}\right) \bar{e}\right)^{T}\left(H^{-1} \otimes I_{n}\right)\left(\left(M_{D} \otimes I_{n}\right) \bar{e}\right)\right]+\left(\left(M_{D} \otimes I_{n}\right) \bar{e}\right)^{T}\left[\left(D \otimes I_{n}\right)\left(\widehat{v}-\left(D \otimes I_{n}\right)\left(1_{N} \otimes v_{0}\right)\right)-c_{1} \operatorname{sgn}\left(\left(M_{D} \otimes I_{n}\right) \bar{e}\right)\right] \\
& \leq-\eta_{1}(t) h_{\max }^{-1}\left\|\left(M_{D} \otimes I_{n}\right) \bar{e}\right\|^{2}+h_{\text {max }}^{-1}\|\bar{\varepsilon}\|\left\|\left(M_{D} \otimes I_{n}\right) \bar{e}\right\|-c_{1} h_{\text {max }}^{-1}\left\|\left(M_{D} \otimes I_{n}\right) \bar{e}\right\| \\
& \leq-2 \eta_{1}(t) h_{\text {max }}^{-1} \lambda_{\min }\left(\left(M_{D} H\right) \otimes I_{n}\right) V_{1}(t)+\left(\|\bar{\varepsilon}\| h_{\max }^{-1}-c_{1} h_{\max }^{-1}\right)\left\|\left(M_{D} \otimes I_{n}\right) \bar{e}\right\| \leq-\frac{\dot{\xi}_{1}(t)}{1-\xi_{1}(t)+\delta_{1}} V_{1}(t) .
\end{aligned}
$$


It thus follows from (22) that $\left\|\widehat{\varepsilon}_{i}(t)\right\|$ $\leq 1 / N \sqrt{2 \delta_{2} / z_{\min }\left(1+\delta_{2}\right) V_{2}(0)}, \forall t>T_{f_{2}}$, and the practical convergence error is disposed by sign function. For $t \in\left[T_{f_{2}}, T_{f_{1}}+T_{f_{2}}\right]$, using Lemma 3 , one draws a conclusion that $V_{1}(t)$ decreases to a set of residuals $\Omega_{1}=\left\{V_{1}(t)\right.$ $\left.\mid V_{1}(t) \leq \delta_{1} / 1+\delta_{1} V_{1}(0)\right\}$ at $T_{f_{1}}+T_{f_{2}}$ that can be attained independent with initial states. Furthermore, one obtains that $\quad \lim _{t \longrightarrow T_{f_{1}}+T_{f_{2}}}\left\|\bar{e}_{i}(t)\right\| \leq 1 / N \sqrt{2 \delta_{1} / z_{\min }\left(1+\delta_{1}\right) V_{1}(0)}$, which is equal to $\lim _{t \longrightarrow T_{f_{1}}+T_{f_{2}}}\left\|\widehat{e}_{i}(t)\right\| \leq 1 / N \sqrt{2 \delta_{1} / z_{\min }(1+}$ $\left.\delta_{1}\right) V_{1}(0)$. This means that the position state error can be predesigned as a required level so long as an appropriate $\delta_{1}$ is applied in $\eta_{1}(t)$. Hence, $\left\|\widehat{e}_{i}(t)\right\| \leq 1 / N \sqrt{2 \delta_{1} / z_{\min }\left(1+\delta_{1}\right) V_{1}(}$ $0), \forall t>T_{f_{1}}+T_{f_{2}}$ and $\lim _{t \longrightarrow+\infty}\left\|\widehat{e}_{i}(t)\right\|=0$. That is, $\widehat{q}_{i} \longrightarrow q_{0}$, $\forall i \in \mathscr{V}^{+} ; \widehat{q}_{i} \longrightarrow-q_{0}, \forall i \in \mathscr{V}^{-}$. This completes the proof.

Remark 4. From Lemma 3, it obtains that the solution of (2) is $x(t)=x_{0}(1-\xi(t) / 1+\delta)$. According to property of $\xi(t)$, it implies that $\xi\left(t_{f}\right)=1$; it thus obtains that $\lim _{t \rightarrow t_{f}}|x(t)| \leq \delta / 1+\delta x_{0}$. When $\delta$ is small enough, the influence of the initial state is negligible. It thus implies that $x(t)=0$ when $t \geq t_{f}$, namely, the fixed-time convergence can be achieved by using the TBG-based approach.

Remark 5. It is worth noting that the closed-loop system $s_{i}=\dot{q}_{i}-\widehat{v}_{i}+\delta_{r}+K_{1 i} \phi\left(\tilde{q}_{i}\right)+K_{2 i} \operatorname{sig}^{l}\left(\widetilde{q}_{i}\right)$, where $\delta_{r}$ is the tiny error in the convergence procedure. Based on the above analysis, $\dot{\hat{q}}_{i}$ can be approximately replaced by $\widehat{v}_{i}$ as $t>T_{f_{1}}+T_{f_{2}}$. It is unnecessary that we employ $\delta_{r}$ in the stability analysis. Therefore, we employ the simplified closed-loop system $s_{i}=\dot{q}_{i}-\widehat{v}_{i}+K_{1 i} \phi\left(\tilde{q}_{i}\right)+K_{2 i} \operatorname{sig}^{l}\left(\widetilde{q}_{i}\right)$ in the subsequent analysis for avoiding redundant proof.

3.3. Stability Analysis for the Fixed-Time Practical Bipartite Tracking for the NRSs. In this section, the stability analysis for the proposed fixed-time estimator-based control algorithm is studied.

Theorem 2. Considering the robot dynamic system with parametric uncertainties and input disturbances described by (4), the input torque $\tau_{i}$ ensures that $\tilde{q}_{i}$ and $\dot{\tilde{q}}_{i}$ globally converge to an user-defined small set $\Delta$ within a fixed time $T_{s}+T_{e}$, where $T_{s}$ and $T_{e}$ is derived as follows:

$$
\begin{aligned}
& T_{s} \leq \frac{2}{\sqrt{m_{1}+m_{2}}}\left(\frac{1}{k}+\frac{n^{(r-1) / 2}}{(r-1) \lambda_{\min }\left(K_{0 i}\right) \sqrt{\left(m_{1}+m_{2}\right)^{r}}}\right), \\
& T_{e} \leq \frac{2^{(1-p) / 2}}{(1-p) \lambda_{\min }\left(K_{1 i}\right)}+\frac{(2 n)^{1-l / 2}}{(l-1) \lambda_{\min }\left(K_{2 i}\right)} .
\end{aligned}
$$

This implies that the fixed-time practical bipartite tracking problem can be solved in the fixed time $T \leq T_{s}+T_{e}+T_{f_{1}}+T_{f_{2}}$, where $T_{f 1}$ and $T_{f 2}$ are user-defined parameters, as presented in Theorem 1 .

Proof. The Lyapunov function candidate was chosen as follows:

$$
V=\frac{1}{2} s_{i}^{T} M_{i 0}\left(q_{i}\right) s_{i}
$$

Taking the derivative of $\mathrm{V}$ with respect to time along (16), we obtain

$$
\dot{V}=s_{i}^{T} M_{i 0}\left(q_{i}\right) \dot{s}_{i} .
$$

After substituting $\dot{s}_{i}$ from the first equation of (17) into (28),

$$
\begin{aligned}
\dot{V} & =s_{i}^{T}\left(-\frac{k+b_{0}+b_{1}\left\|\dot{q}_{i}\right\|^{2}+b_{2}\left\|\tau_{i 0}+\tau_{i 1}\right\|}{\left(1-b_{2}\right)\left\|s_{i}\right\|} s_{i}+\rho-K_{0 i} \operatorname{sig}^{r}\left(s_{i}\right)\right) \\
& =-\frac{k+b_{0}+b_{1}\left\|\dot{q}_{i}\right\|^{2}+b_{2}\left\|\tau_{i 0}+\tau_{i 1}\right\|}{\left(1-b_{2}\right)\left\|s_{i}\right\|} s_{i}^{T} s_{i}+s_{i}^{T} \rho-s_{i}^{T} K_{0 i} \operatorname{sig}^{r}\left(s_{i}\right) \\
& =-\frac{k+b_{0}+b_{1}\left\|\dot{q}_{i}\right\|^{2}+b_{2}\left\|\tau_{i 0}+\tau_{i 1}\right\|}{1-b_{2}}\left\|s_{i}\right\|+s_{i}^{T} \rho-s_{i}^{T} K_{0 i} \operatorname{sig}^{r}\left(s_{i}\right) \\
& =-b_{2}\left\|s_{i}\right\| \tau_{\text {aux }}-\left\|s_{i}\right\|\left(k+b_{0}+b_{1}\left\|\dot{q}_{i}\right\|^{2}+b_{2}\left\|\tau_{i 0}+\tau_{i 1}\right\|\right)+s_{i}^{T} \rho-s_{i}^{T} K_{0 i} \operatorname{sig}^{r}\left(s_{i}\right) \\
& \leq-b_{2}\left\|s_{i}\right\| \tau_{\text {aux }}-\left\|s_{i}\right\|\left(k+b_{0}+b_{1}\left\|\dot{q}_{i}\right\|^{2}+b_{2}\left\|\tau_{i 0}+\tau_{i 1}\right\|\right)+\left\|s_{i}\right\|\|\rho\|-s_{i}^{T} K_{0 i} \operatorname{sig}^{r}\left(s_{i}\right) .
\end{aligned}
$$


Denote $s_{i j}$ as the $j$ th element of the $s_{i}$; substituting (7) into (29), it follows that

$$
\begin{aligned}
\dot{V} & \leq-b_{2}\left\|s_{i}\right\| \tau_{\text {aux }}-\left\|s_{i}\right\|\left(k+b_{0}+b_{1}\left\|\dot{q}_{i}\right\|^{2}+b_{2}\left\|\tau_{i 0}+\tau_{i 1}\right\|\right)+\left\|s_{i}\right\|\left(b_{0}+b_{1}\left\|\dot{q}_{i}\right\|^{2}+b_{2}\left\|\tau_{i}\right\|\right)-s_{i}^{T} K_{0 i} \operatorname{sig}^{r}\left(s_{i}\right) \\
& \leq-s_{i}^{T} K_{0 i} \operatorname{sig}^{r}\left(s_{i}\right)-k\left\|s_{i}\right\|-b_{2}\left\|s_{i}\right\| \tau_{a u x}+b_{2}\left\|s_{i}\right\|\left\|\tau_{i 2}\right\| \\
& \leq-s_{i}^{T} K_{0 i} \operatorname{sig}^{r}\left(s_{i}\right)-k\left\|s_{i}\right\| \\
& \leq-\lambda_{\min }\left(K_{0 i}\right) \sum_{j=1}^{n}\left|s_{i j}\right|^{r+1}-k\left\|s_{i}\right\| \\
& \leq-n^{(1-r) / 2} \lambda_{\min }\left(K_{0 i}\right)\left\|s_{i}\right\|^{r+1}-k\left\|s_{i}\right\| .
\end{aligned}
$$

Suppose that $m_{1} \leq\left\|M_{i 0}^{-1}\left(q_{i}\right)\right\| \leq m_{2}$. Choosing $M_{i 0}=2 /$ $m_{1}+m_{2} I_{n}[45]$, we have

$$
V=\frac{1}{m_{1}+m_{2}}\left\|s_{i}\right\|^{2} .
$$

Applying (31) to (30), it follows that

$$
\dot{V}+k \sqrt{m_{1}+m_{2}} \sqrt{V}+n^{(1-r) / 2} \lambda_{\min }\left(K_{0 i}\right)\left(m_{1}+m_{2}\right)^{(r+1) / 2} V^{(r+1) / 2} \leq 0 .
$$

$$
\begin{aligned}
\dot{V}_{c a 1} & =\tilde{q}_{i}^{T} \dot{\tilde{q}}_{i} \\
& =-\tilde{q}_{i}^{T} K_{1 i} \operatorname{sig}^{p}\left(\tilde{q}_{i}\right)-\tilde{q}_{i}^{T} K_{2 i} \operatorname{sig}^{l}\left(\tilde{q}_{i}\right) \\
& \leq-\lambda_{\min }\left(K_{1 i}\right)\left\|\tilde{q}_{i}\right\|^{p+1}-n^{(1-l) / 2} \lambda_{\min }\left(K_{2 i}\right)\left\|\tilde{q}_{i}\right\|^{l+1} \\
& \leq-2^{(p+1) / 2} \lambda_{\min }\left(K_{1 i}\right) V_{c a 1}^{(p+1) / 2}-2^{(1+l) / 2} n^{(1-l) / 2} \lambda_{\min }\left(K_{2 i}\right) V_{c a 1}^{(1+l) / 2}
\end{aligned}
$$

Invoking Lemma 4 again, the tracking error $\tilde{q}$ converges to the arbitrary small $\Delta$ within the time $T_{e}$ given by (26).

Case $2\left(\left\|\tilde{q}_{i}\right\|<\Delta\right)$ : applyịng (10), the dynamics can be definitely simplified as $\dot{\vec{q}}_{i}=-p \Delta^{p-1} K_{1 i} \widetilde{q}_{i}-K_{2 i} \operatorname{sig}^{l}\left(\widetilde{q}_{i}\right)$. Selecting the appropriate Lyapunov function candidate defined by Case 1, we obtain

$$
\dot{V}_{c a 2}=-p \Delta^{p-1} \tilde{q}_{i}^{T} K_{1 i} \tilde{q}_{i}-\tilde{q}_{i}^{T} K_{2 i} \operatorname{sig}\left(\tilde{q}_{i}\right) \leq-2 p \Delta^{p-1} \lambda_{\min }\left(K_{1 i}\right) V_{c a 2} .
$$

It is obvious seen that the tracking errors $\tilde{q}_{i}$ exponentially converge to zero.

Remark 6. It is of great significance that we introduce a linear term in the function $\phi\left(\tilde{q}_{i}\right)$ for the case of $\left\|\tilde{q}_{i}\right\|<\Delta$. Otherwise, the control design with $\phi\left(\tilde{q}_{i}\right)$ may generate singularity problem due to the first derivative of $\phi\left(\tilde{q}_{i}\right)$ including a negative power $(p-1)$ on $\tilde{q}_{i}$, which may go to infinity while $x$ goes to zero. This may lead to the desired control cost infinity.
By Lemma 4 , it obtains that $s_{i}$ converge to zero when $t \longrightarrow T_{s}$, which is predefined in (25). After the sliding surface $s_{i}=0$ is achieved within $T_{s}$, the system dynamics are converted to $\tilde{q}_{i}=-K_{1 i} \phi\left(\tilde{q}_{i}\right)-K_{2 i} \operatorname{sig}^{l}\left(\tilde{q}_{i}\right)$. Then, the convergence of $\tilde{q}_{i}$ can be settled by the following two cases.

Case $1\left(\left\|\tilde{q}_{i}\right\|>\Delta\right)$ : the dynamics can be explicitly simplified as $\dot{\tilde{q}}_{i}=-K_{1 i} \operatorname{sig}^{p}\left(\tilde{q}_{i}\right)-K_{2 i} \operatorname{sig}^{l}\left(\tilde{q}_{i}\right)$. Choose the Lyapunov function as follows: $V_{c a 1}=1 / 2 \tilde{q}_{i}^{T} \widetilde{q}_{i}$; the time derivation of $V_{c a l}$ is
Remark 7. The term of $-\left(\eta_{1}(t)+1\right)\left(\sum_{j=1}^{N}\left|a_{i j}\right|\left(\hat{q}_{i}(t)-\right.\right.$ $\left.\left.\operatorname{sgn}\left(a_{i j}\right) \widehat{q}_{j}(t)\right)+a_{i 0}\left(\widehat{q}_{i}-d_{i} q_{0}\right)\right)$ in protocol (13) is TBGbased, which guarantees that the position and velocity error finally converge to a required boundary within the prescribed time. The TBG-based protocol generates a benign margin for convergence time, which has practicability in projects.

\section{Simulation Results}

In this section, simulation experiments are carried out to verify the effectiveness of the proposed algorithm. Assume that the NRS contains eight two-DOF robotic manipulators with interactions displayed in Figure 3.

The communication mode includes one leader and two subnetworks. There only exist cooperative relationships among the robots in each subnetwork by the solid lines, while there are the competitive relationships between the two subnetworks by the dotted lines. Besides, there are an even number of negative weights in each loop. It thus obtained that Assumption 1 holds. In addition, bidirection communication between two vertexes stands for different information delivered between two robots. 


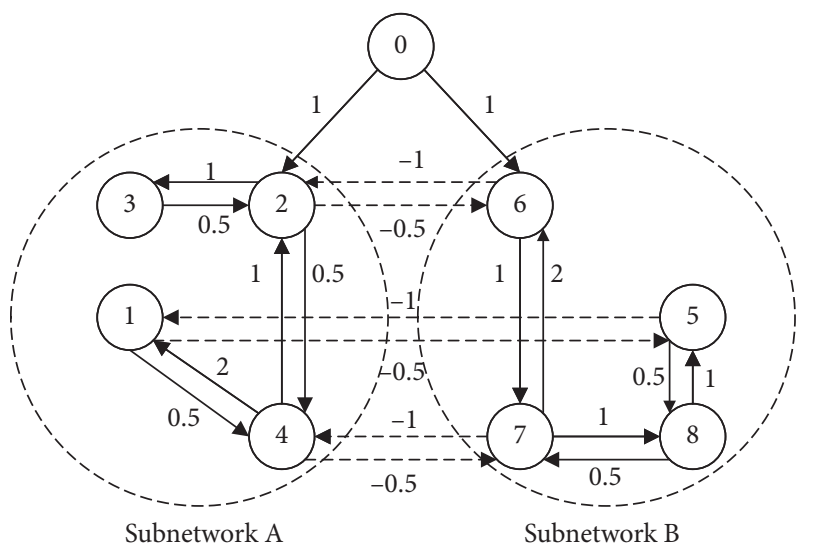

Figure 3: The directed signed graph.

Furthermore, we propose a simple application of our control strategy, in which two parts of robots cooperate and compete with each other to avoid obstacles in the task. In the phase 1 , the robots cooperate with each other and track the target before detecting the obstacle. In the phase 2 , when the robots detect obstacles, the eight robots which have both cooperative and competitive relationships can be split into two clusters to bypass the obstacle. In the phase 3 , the robots cooperate with each other and track the target again after passing the obstacle.

In the practical application, the communication data are determined by the hardware setting of the communication system. However, in the theoretical research, we need to employ the communication data of the directed signed graph to verify the effective of the control algorithm.

The physical parameters of the eight robotic manipulators are listed in Table 1 , where $m_{k}, k \in\{1,2\}$, stands for the masses of links, $l_{k}$ represents the lengths of links, $r_{k}$ is the center of the link's mass, and $J_{k}$ represents the moment of inertia of links.

The initial conditions of robots are chosen as $q(0)=\left[q_{10}^{T}, q_{20}^{T}\right]^{T}, v(0)=\left[v_{10}^{T}, v_{20}^{T}\right]^{T}, \widehat{q}(0)=\left[\hat{q}_{10}^{T}, \hat{q}_{20}^{T}\right]^{T}$, and $\widehat{v}(0)=\left[\widehat{v}_{10}^{T}, \widehat{v}_{20}^{T}\right]^{T}$, where

$$
\begin{aligned}
& q_{10}=[3,-2,-2,3,-2,-2,3,-2]^{T}, \\
& q_{20}=[-2,3,-1,2,3,-1,-2,3]^{T}, \\
& v_{10}=[-1,-3,2,-1,-3,2,-1,-3]^{T}, \\
& v_{20}=[-3,6,-5,-7,3,1,-2,7]^{T}, \\
& \widehat{q}_{10}=[1,2,-3,1,2,-3,1,2]^{T}, \\
& \widehat{q}_{20}=[-1,8,5,-7,1,-3,2,4]^{T}, \\
& \widehat{v}_{10}=[-1,-3,2,-1,-3,2,-1,-3]^{T}, \\
& \widehat{v}_{20}=[2,2,-3,2,2,-3,2,2]^{T} .
\end{aligned}
$$

The position, velocity, and acceleration states of the leader are, respectively, selected as $q_{0}=[\sin (t) ; \sin (t)]^{T}$, $v_{0}=[\cos (t) ; \cos (t)]^{T}$, and $u_{0}=[-\sin (t) ;-\sin (t)]^{T}$. The control parameters are given as $p=0.5, q=1.2, r=1.5, k=$ $1, b_{0}=12, b_{1}=2.8, b_{2}=0.5, \delta_{1}=\delta_{2}=0.01, c_{1}=0.01, c_{2}=1$,
TABLE 1: The physical parameters of the eight robotic manipulators.

\begin{tabular}{lcccc}
\hline$i$ th robot & $m_{k}(\mathrm{~kg})$ & $l_{k}(m)$ & $r_{k}(m)$ & $J_{k}\left(\mathrm{~kg} \cdot \mathrm{m}^{2}\right)$ \\
\hline 1 & $1.32,1.08$ & $2.76,2.48$ & $1.38,1.24$ & $0.84,0.55$ \\
2 & $1.23,1.02$ & $2.64,2.42$ & $1.32,1.21$ & $0.71,0.50$ \\
3 & $1.26,1.04$ & $2.68,2.44$ & $1.34,1.22$ & $0.75,0.52$ \\
4 & $1.32,1.08$ & $2.76,2.48$ & $1.38,1.24$ & $0.84,0.55$ \\
5 & $1.23,1.02$ & $2.64,2.42$ & $1.32,1.21$ & $0.71,0.50$ \\
6 & $1.26,1.04$ & $2.68,2.44$ & $1.34,1.22$ & $0.75,0.52$ \\
7 & $1.32,1.08$ & $2.76,2.48$ & $1.38,1.24$ & $0.84,0.55$ \\
8 & $1.23,1.02$ & $2.64,2.42$ & $1.32,1.21$ & $0.71,0.50$ \\
\hline
\end{tabular}

$K_{0 i}=\operatorname{diag}(4,3), K_{1 i}=\operatorname{diag}(2,2)$, and $K_{2 i}=\operatorname{diag}(2,2)$. The input disturbance $d_{i}(t)=0.1[\cos (t) ; \sin (t)]^{T}$. From Figure 1, the Laplacian matrix $L_{s}$ is

$$
L_{s}=\left[\begin{array}{cccccccc}
3 & 0 & 0 & -2 & 1 & 0 & 0 & 0 \\
0 & 2.5 & -0.5 & -1 & 0 & 1 & 0 & 0 \\
0 & -1 & 1 & 0 & 0 & 0 & 0 & 0 \\
-0.5 & -0.5 & 0 & 2 & 0 & 0 & 1 & 0 \\
0.5 & 0 & 0 & 0 & 1.5 & 0 & 0 & -1 \\
0 & 0.5 & 0 & 0 & 0 & 2.5 & -2 & 0 \\
0 & 0 & 0 & 0.5 & 0 & -1 & 2 & -0.5 \\
0 & 0 & 0 & 0 & -0.5 & 0 & -1 & 1.5
\end{array}\right],
$$

where $\quad B=\operatorname{diag}(0,1,0,0,0,1,0,0) \quad$ and $\quad H=\operatorname{diag}$ $(2,1,2,0.5,1,0.5,0.25,0.5)$.

Based on the aforementioned parameter setting, the simulation results of the proposed algorithms are shown in Figures 4-9. From Figures 4 and 5, it is shown that the robots' position and velocity states are divided into two groups to track the leader's trajectory, where robots belonging to subnetwork A converge to the states of the leader within the predesigned time, while the robots belonging to subnetwork B approach to the opposite state of the leader within the predesigned time. In Figures 6-8, the tracking errors are, respectively, provided to illustrated the tracking performances of the position states. From Figure 7, the 

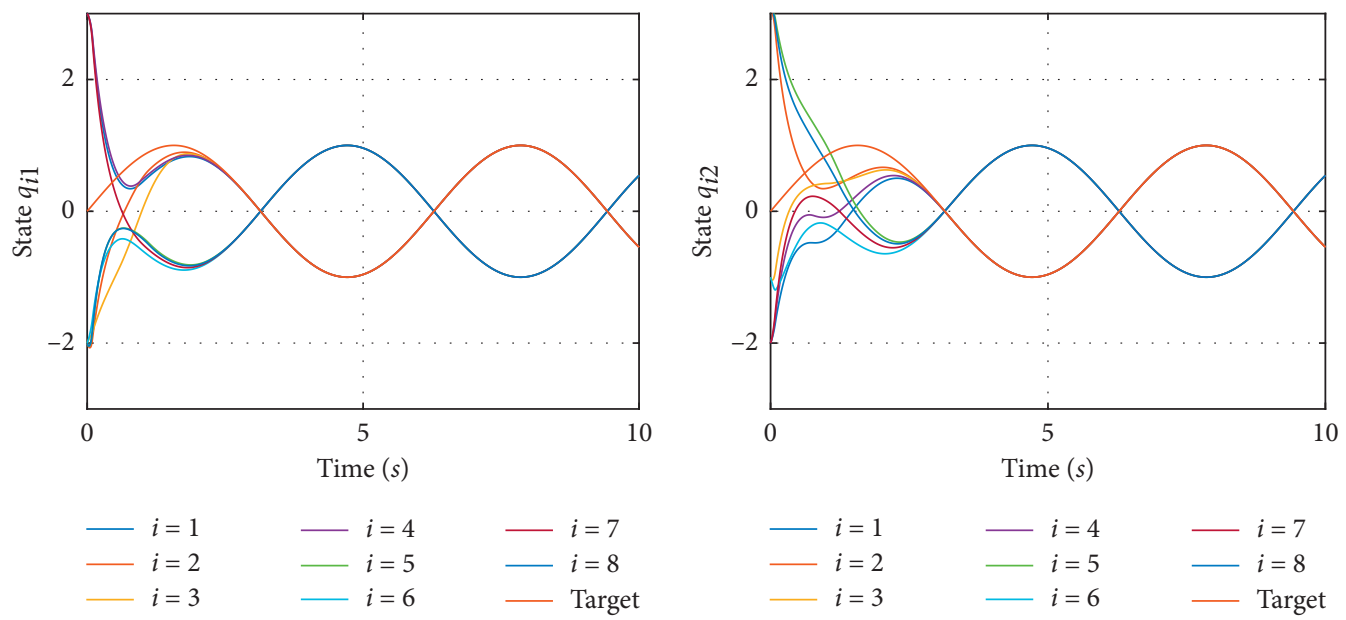

(a)

(b)

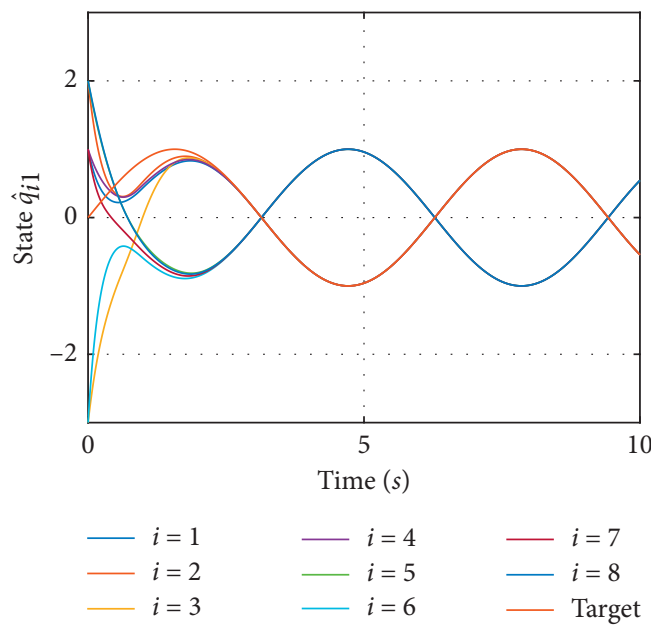

(c)

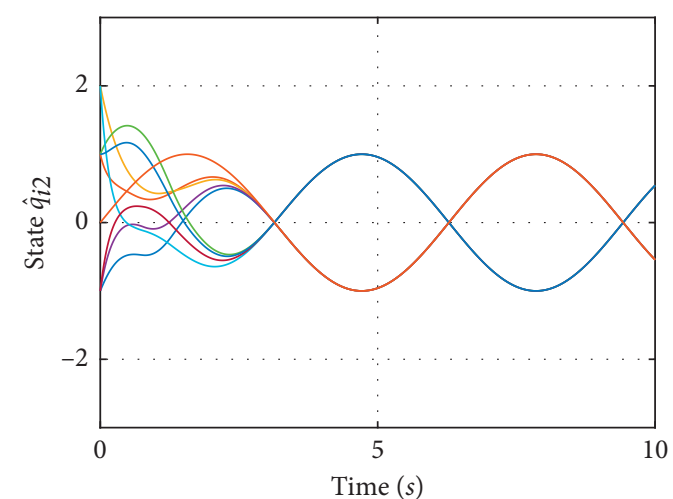

$i=4 \quad i=7$

$-i=5 \quad-i=8$

(d)

FIGURE 4: Pictures (a) and (b) reveal the evolution of $q_{i}$ for links 1 and 2, respectively. Pictures (c) and (d) reveal the evolution of $\widehat{q}_{i}$ for links 1 and 2 , respectively.
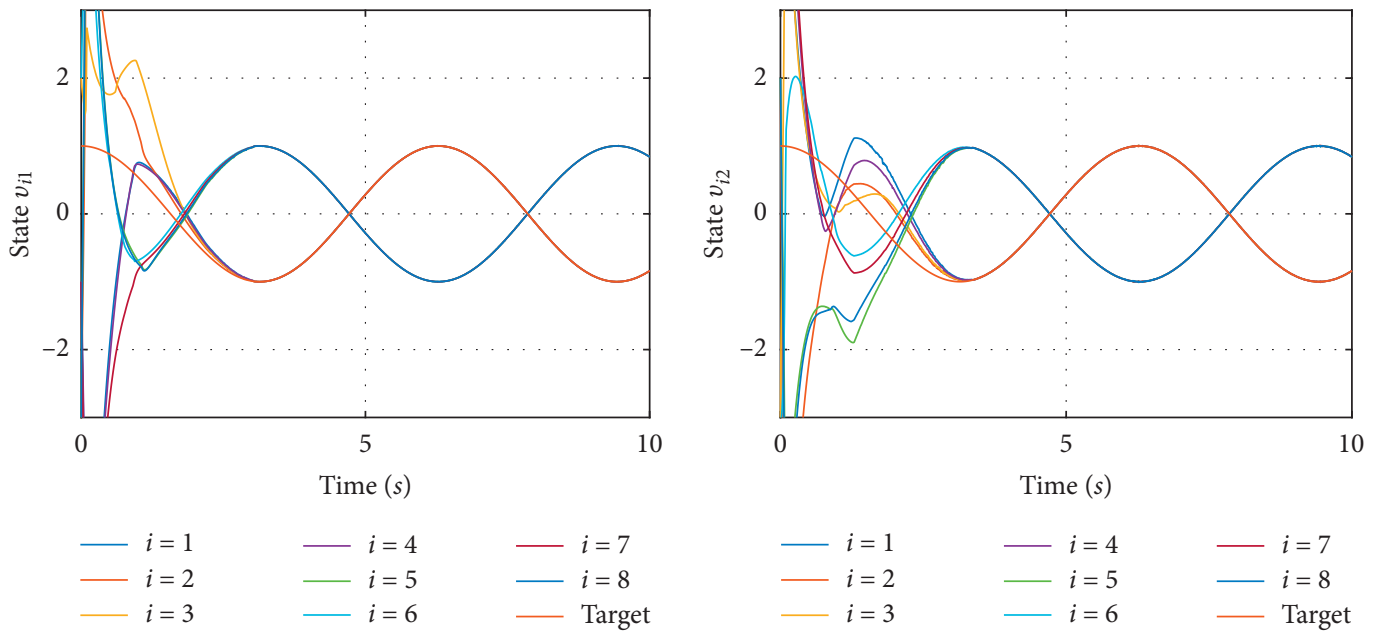

(a)

Figure 5: Continued.

(b) 


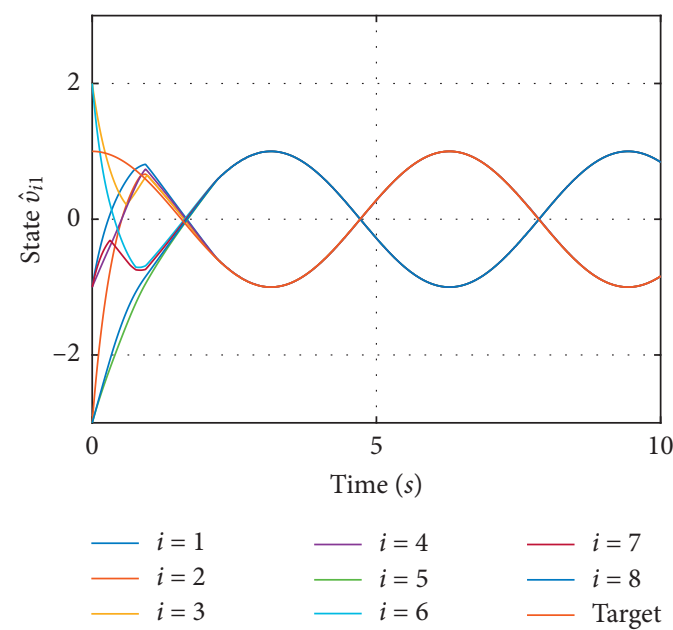

(c)

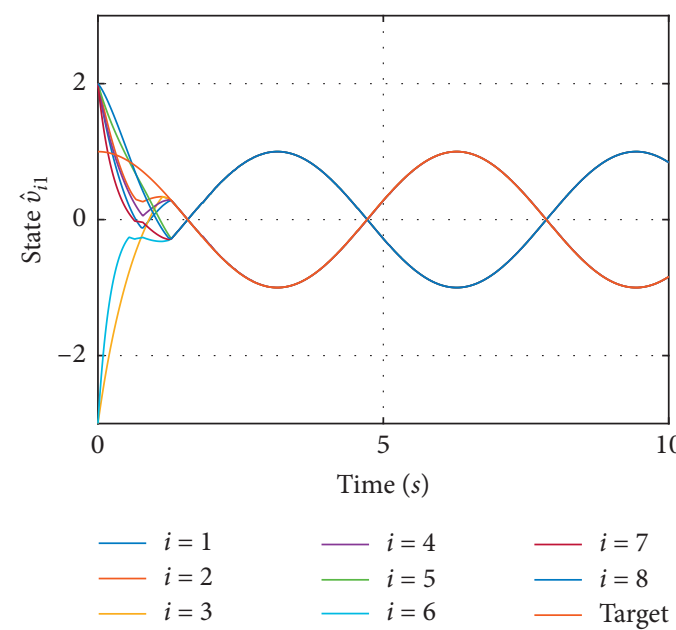

(d)

Figure 5: Pictures (a) and (b) reveal the evolution of $v_{i}$ for links 1 and 2, respectively. Pictures (c) and (d) reveal the evolution of $\widehat{v}_{i}$ for links 1 and 2 , respectively.
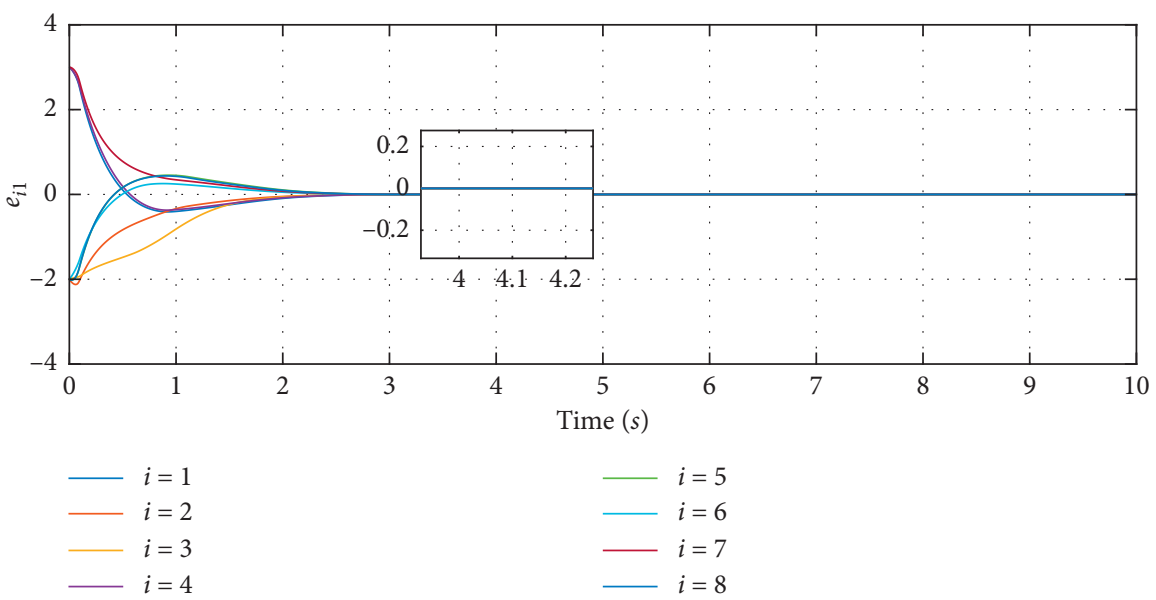

(a)

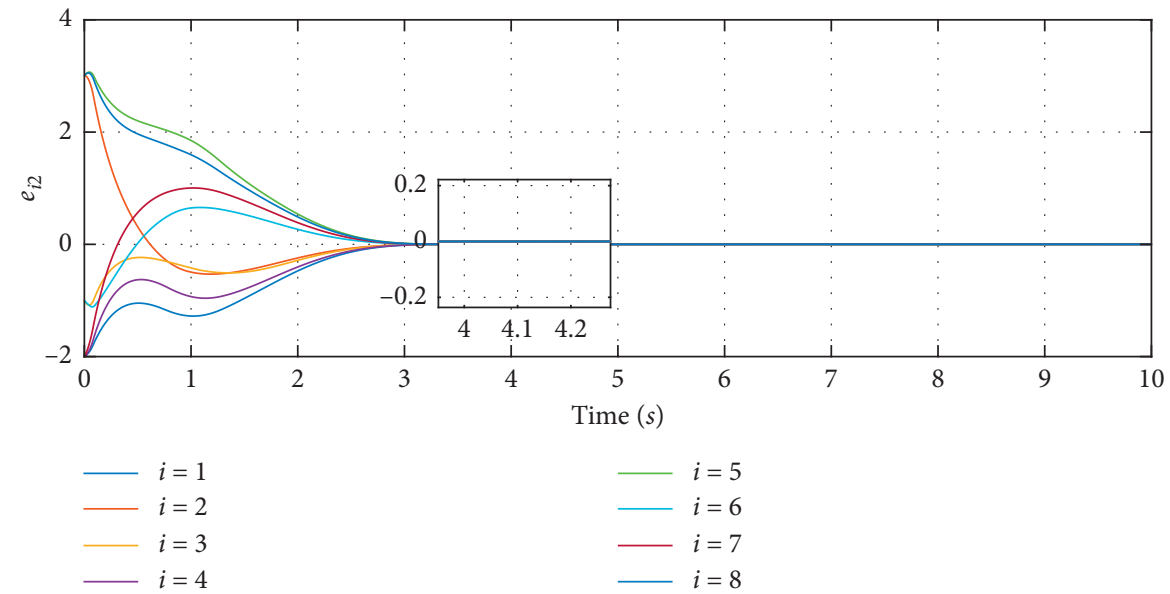

(b)

Figure 6: Pictures (a) and (b) reveal the evolution of the position tracking errors for links 1 and 2. 


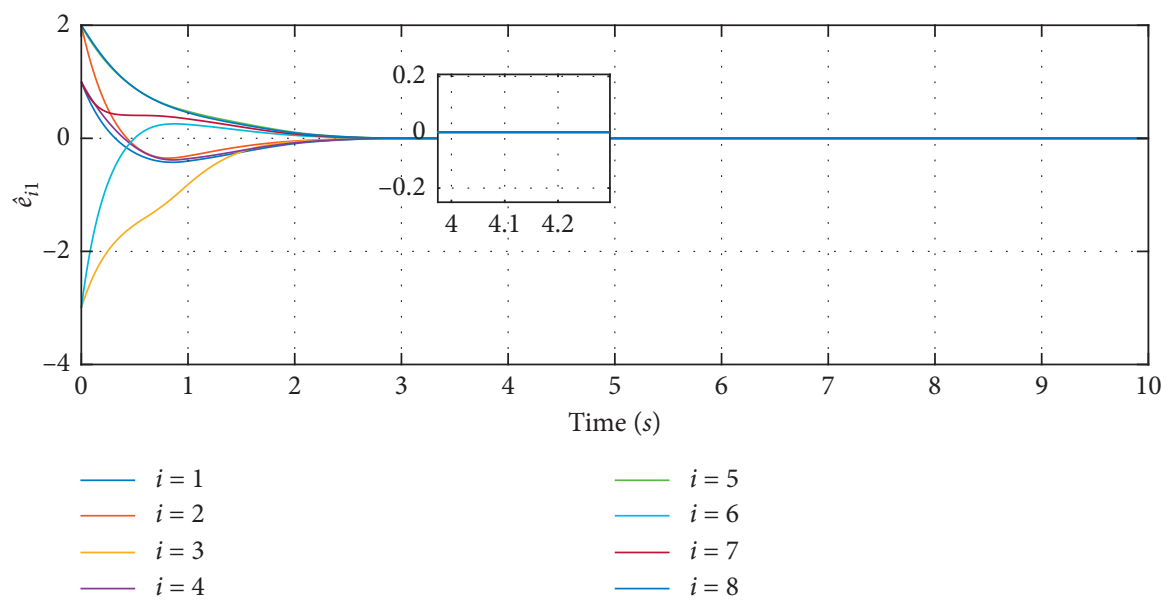

(a)
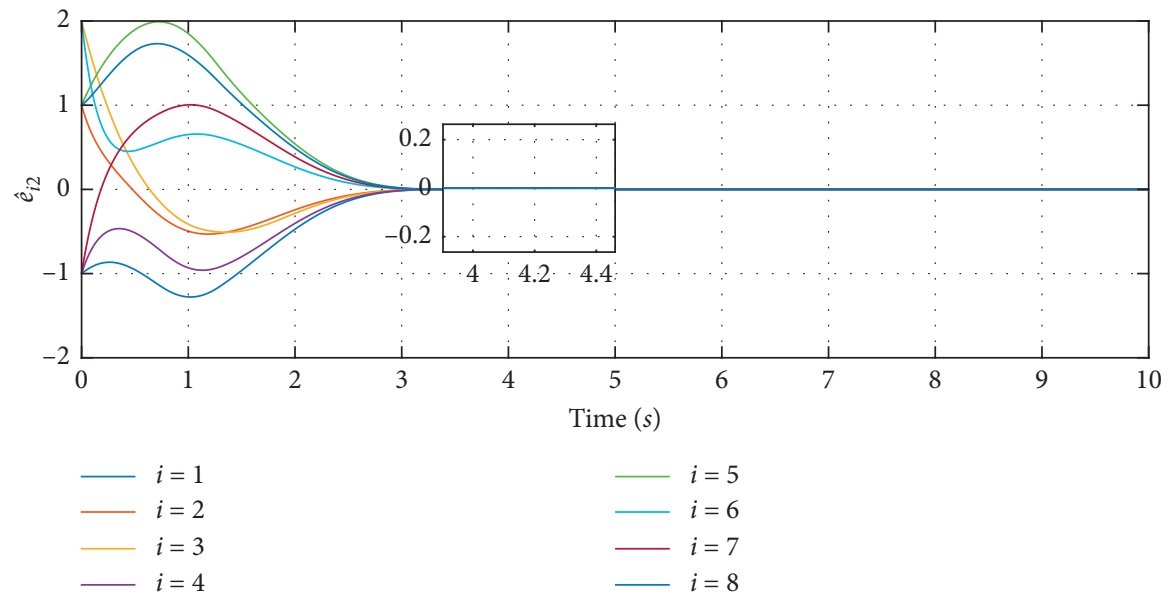

(b)

Figure 7: Pictures (a) and (b) reveal the evolution of the errors $\widehat{e}_{i}$ for links 1 and 2.

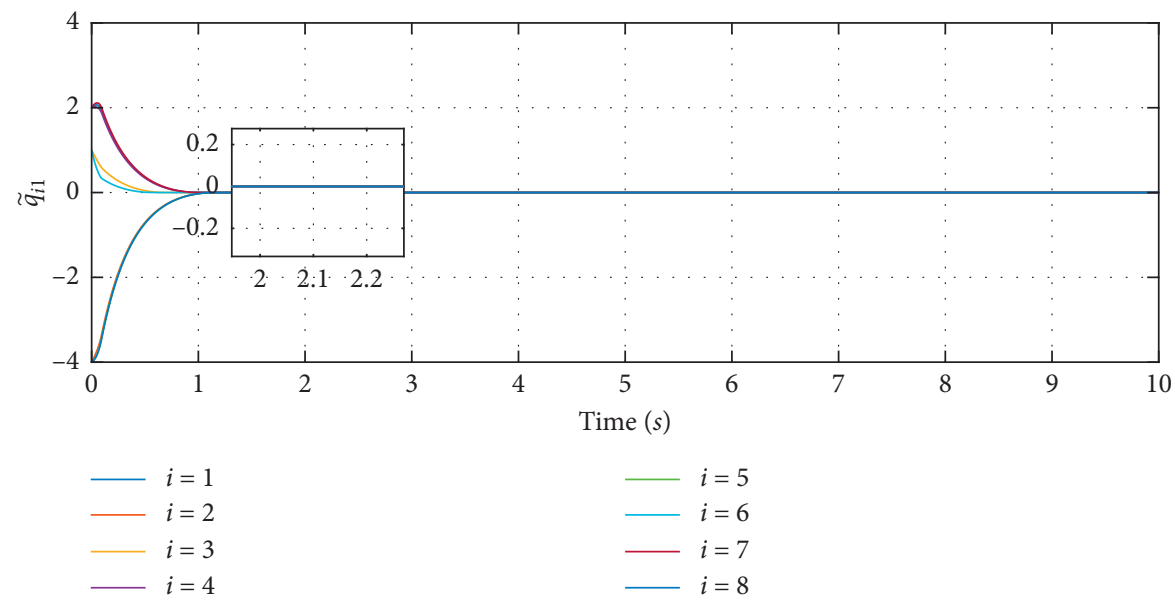

(a)

Figure 8: Continued. 


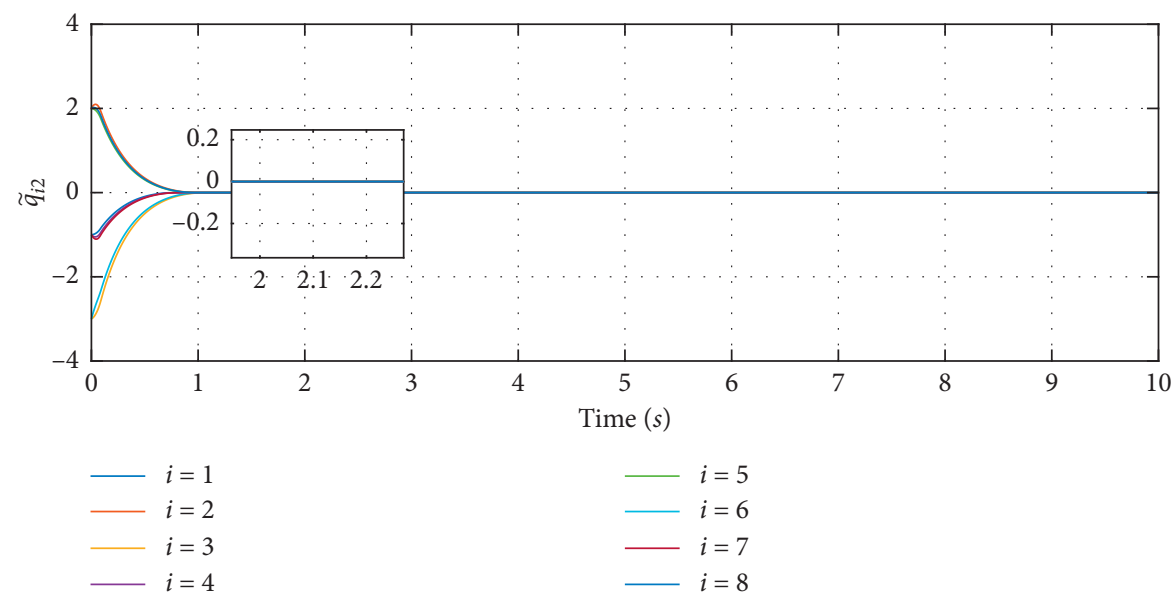

(b)

Figure 8: Pictures (a) and (b) reveal the evolution of the errors $\tilde{q}_{i}$ for links 1 and 2.

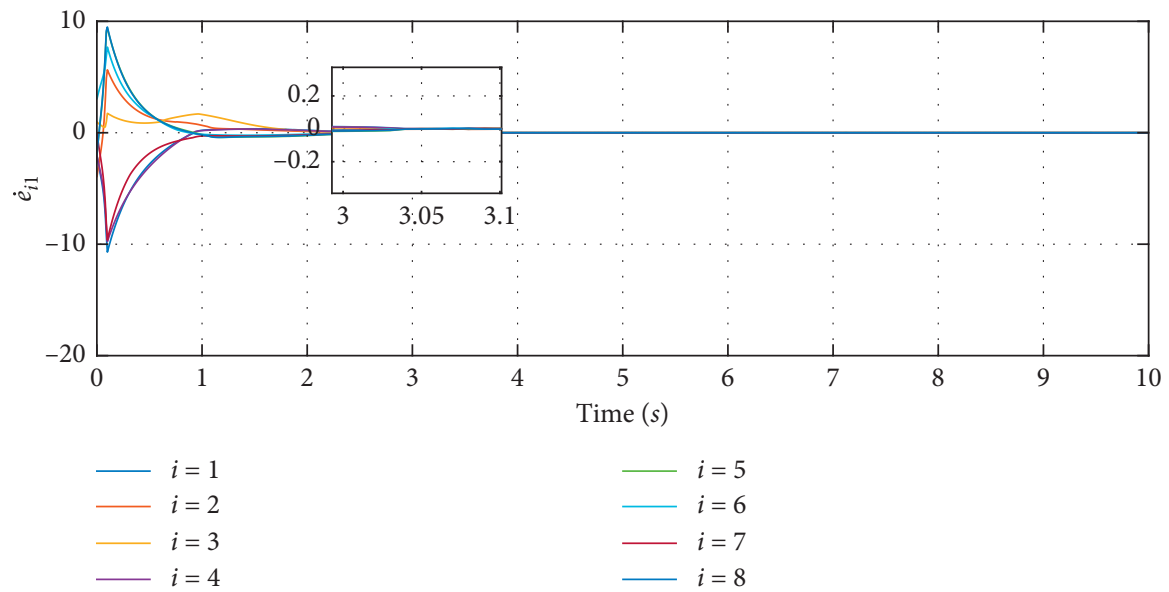

(a)

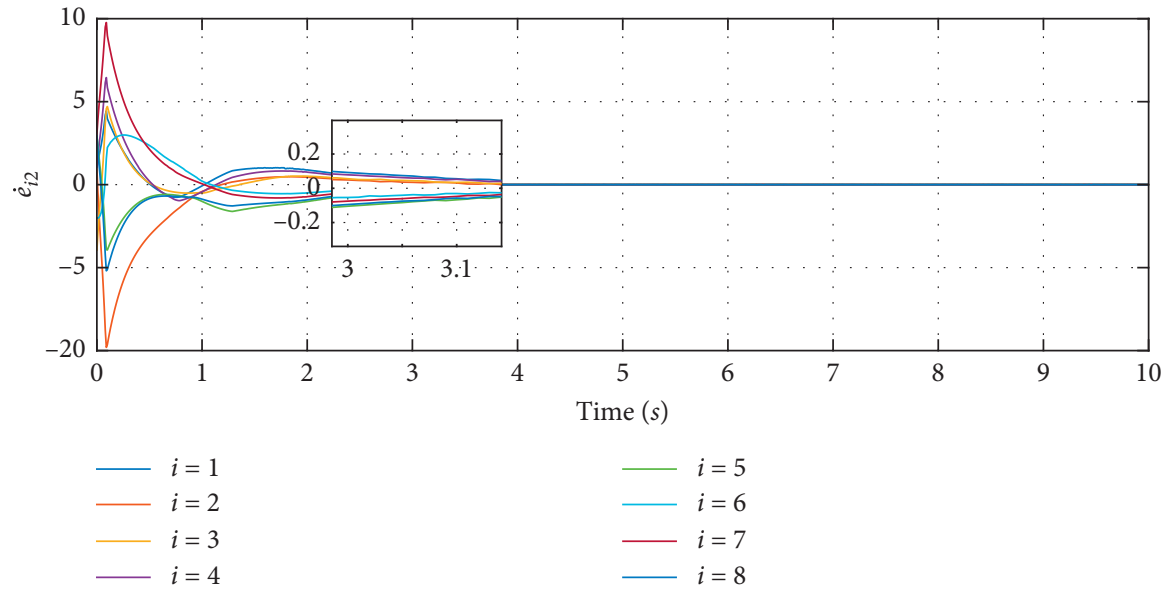

(b)

FIgURe 9: Pictures (a) and (b) reveal the evolution of the velocity tracking errors for links 1 and 2.

estimators $\widehat{q}_{i}$ can track the neighbourhood of leader state $q_{0}$ within $T_{f_{1}}+T_{f_{2}}$, which proves the effectiveness of the estimator layer. From Figure 8 , the true value $q_{i}$ can converge to the neighbourhood of estimators $\widehat{q}_{i}$ within $T_{s}+T_{e}$, which demonstrates the effectiveness of the local control layer. In Figures 6 and 9, the tracking errors $e_{i}$ and $\dot{e}_{i}$ converge to the 
neighbourhood of the origin within the convergence time $T$, which indicate the effectiveness of the proposed algorithms.

\section{Conclusion}

In this paper, the fixed-time practical bipartite tracking problem for the NRSs has been investigated under parametric uncertainties, input disturbances, and directed signed graph. A new fixed-time estimator-based control algorithm is proposed to solve this problem. Some necessary conditions are established. The validity of the proposed algorithm was demonstrated in the simulation graph. Future work will concentrate on bipartite tracking problem with uniform time delay and input saturation.

\section{Data Availability}

No data were used to support this study.

\section{Conflicts of Interest}

The authors declare that they have no conflicts of interest.

\section{Acknowledgments}

This work was supported by the National Natural Science Foundation of China under Grants 51805494, 61703374, 51805496, and 62073301 and the Fundamental Research Founds for National University and China University of Geosciences (Wuhan) under Grant 1910491B05.

\section{References}

[1] H. Li, Z. Chen, Y. Sun, and H. R. Karimi, "Stabilization for a class of nonlinear networked control systems via polynomial fuzzy model approach," Complexity, vol. 21, no. 2, pp. 74-81, 2015.

[2] H. Zhao and J. H. Park, "Dynamic output feedback consensus of continuous-time networked multiagent systems," Complexity, vol. 20, no. 5, pp. 35-42, 2015.

[3] M.-F. Ge, Z.-W. Liu, G. Wen, X. Yu, and T. Huang, "Hierarchical controller-estimator for coordination of networked Euler-Lagrange systems," IEEE Transactions on Cybernetics, vol. 50, no. 6, pp. 2450-2461, 2020.

[4] X.-S. Zhan, L.-L. Cheng, J. Wu, and H.-C. Yan, "Modified tracking performance limitation of networked time-delay systems with two-channel constraints," Journal of the Franklin Institute, vol. 356, no. 12, pp. 6401-6418, 2019.

[5] M.-F. Ge, Z.-H. Guan, B. Hu, D.-X. He, and R.-Q. Liao, "Distributed controller-estimator for target tracking of networked robotic systems under sampled interaction," Automatica, vol. 69, pp. 410-417, 2016.

[6] H. Wang, Y. Wang, C. Li, J. Li, and X. Ban, "Adaptive weight update algorithm for target tracking of UUV based on improved Gaussian mixture cubature Kalman filter," Complexity, vol. 2020, Article ID 7828050, 2020.

[7] C.-D. Liang, M.-F. Ge, Z.-W. Liu, G. Ling, and F. Liu, "Predefined-time formation tracking control of networked marine surface vehicles," Control Engineering Practice, vol. 107, Article ID 104682, 2021.

[8] M. F. Ge, C. D. Liang, X. S. Zhan, C. Y. Chen, G. Xu, and J. Chen, "Multiple time-varying formation of networked heterogeneous robotic systems via estimator-based hierarchical cooperative algorithms," Complexity, vol. 2020, Article ID 8357428, 2020.

[9] L. Wang, H. He, Z. Zeng, and M.-F. Ge, "Model-independent formation tracking of multiple euler-Lagrange systems via bounded inputs," IEEE Transactions on Cybernetics, vol. 51, no. 5, pp. 2813-2823, 2021.

[10] X. Li, C. Wen, X. Fang, and J. Wang, “Adaptive bearing-only formation tracking control for nonholonomic multiagent systems," IEEE Transactions on Cybernetics, pp. 1-11, 2021.

[11] C.-D. Liang, M.-F. Ge, Z.-W. Liu, Y.-W. Wang, and H. R. Karimi, "Output multiformation tracking of networked heterogeneous robotic systems via finite-time hierarchical control," IEEE Transactions on Cybernetics, vol. 51, no. 6, pp. 2893-2904, 2021.

[12] X. Wang, S. Li, and P. Shi, "Distributed finite-time containment control for double-integrator multiagent systems," IEEE Transactions on Cybernetics, vol. 44, no. 9, pp. 1518-1528, 2013.

[13] D. Wang, N. Zhang, J. Wang, and W. Wang, "Cooperative containment control of multiagent systems based on follower observers with time delay," IEEE Transactions on Systems, Man, and Cybernetics: Systems, vol. 47, no. 1, pp. 13-23, 2016.

[14] Y.-W. Wang, Y.-W. Wei, X.-K. Liu, N. Zhou, and C. G. Cassandras, "Optimal persistent monitoring using second-order agents with physical constraints," IEEE Transactions on Automatic Control, vol. 64, no. 8, pp. 3239-3252, 2019.

[15] X.-S. Zhan, J. Wu, T. Jiang, and X.-W. Jiang, "Optimal performance of networked control systems under the packet dropouts and channel noise," ISA Transactions, vol. 58, no. 5, pp. 214-221, 2015.

[16] X. Hu, Z.-W. Liu, G. Wen, X. Yu, and C. Li, "Branch-wise parallel successive algorithm for online voltage regulation in distribution networks," IEEE Transactions on Smart Grid, vol. 10, no. 6, pp. 6678-6689, 2019.

[17] Z. W. Liu, G. H. Wen, X. H. Yu, Z. H. Guan, and T. W. Huang, "Delayed impulsive control for consensus of multiagent systems with switching communication graphs," IEEE Transactions on Cybernetics, vol. 50, no. 7, pp. 3045-3055, 2019.

[18] C. Y. Chen, W. H. Gui, L. H. Wu, Z. H. Liu, and H. C. Yan, "Tracking performance limitations of MIMO networked control systems with multiple communication constraints," IEEE Transactions on Cybernetics, vol. 50, no. 7, pp. 29822995, 2019.

[19] C.-Y. Chen, F. Liu, L. Wu, H. Yan, W. Gui, and H. E. Stanley, "Tracking performance limitations of networked control systems with repeated zeros and poles," IEEE Transactions on Automatic Control, vol. 66, no. 4, pp. 1902-1909, 2021.

[20] X. Li, C. Wen, and C. Chen, "Resilient cooperative control for networked Lagrangian systems against DoS attacks," IEEE Transactions on Cybernetics, pp. 1-13, 2020.

[21] Y. Chang, S. Zhang, N. D. Alotaibi, and A. F. Alkhateeb, "Observer-based adaptive finite-time tracking control for a class of switched nonlinear systems with unmodeled dynamics," IEEE Access, vol. 8, pp. 204782-204790, 2020.

[22] Y. Wang, N. Xu, Y. Liu, and X. Zhao, "Adaptive fault-tolerant control for switched nonlinear systems based on command filter technique," Applied Mathematics and Computation, vol. 392, Article ID 125725, 2021.

[23] Y. Wang, Y. Chang, A. F. Alkhateeb, and N. D. Alotaibi, "Adaptive fuzzy output-feedback tracking control for switched nonstrict-feedback nonlinear systems with 
prescribed performance," Circuits, Systems, and Signal Processing, vol. 40, no. 1, pp. 88-113, 2020.

[24] Z.-M. Li, X.-H. Chang, and J. H. Park, "Quantized static output feedback fuzzy tracking control for discrete-time nonlinear networked systems with asynchronous event-triggered constraints," IEEE Transactions on Systems, Man, and Cybernetics: Systems, vol. 51, no. 6, pp. 3820-3831, 2021.

[25] X. Zhao, X. Wang, L. Ma, and G. Zong, "Fuzzy approximation based asymptotic tracking control for a class of uncertain switched nonlinear systems," IEEE Transactions on Fuzzy Systems, vol. 28, no. 4, pp. 632-644, 2019.

[26] C. Altafini, "Consensus problems on networks with antagonistic interactions," IEEE Transactions on Automatic Control, vol. 58, no. 4, pp. 935-946, 2013.

[27] J. Hu, Y. Wu, L. Liu, and G. Feng, "Adaptive bipartite consensus control of high-order multiagent systems on coopetition networks," International Journal of Robust and Nonlinear Control, vol. 28, no. 7, pp. 2868-2886, 2018.

[28] T.-F. Ding, M.-F. Ge, C.-H. Xiong, and J. H. Park, "Bipartite consensus for networked robotic systems with quantized-data interactions," Information Sciences, vol. 511, pp. 229-242, 2020.

[29] D. Meng, "Bipartite containment tracking of signed networks," Automatica, vol. 79, pp. 282-289, 2017.

[30] M. Liu, X. Wang, and Z. Li, "Robust bipartite consensus and tracking control of high-order multiagent systems with matching uncertainties and antagonistic interactions," IEEE Transactions on Systems, Man, and Cybernetics: Systems, vol. 50, no. 7, pp. 2541-2550, 2018.

[31] T.-F. Ding, M.-F. Ge, Z.-W. Liu, Y.-W. Wang, and H. R. Karimi, "Lag-bipartite formation tracking of networked robotic systems over directed matrix-weighted signed graphs," IEEE Transactions on Cybernetics, pp. 1-12, 2020.

[32] G. Wen, H. Wang, X. Yu, and W. Yu, "Bipartite tracking consensus of linear multi-agent systems with a dynamic leader," IEEE Transactions on Circuits and Systems II: Express Briefs, vol. 65, no. 9, pp. 1204-1208, 2017.

[33] A. Polyakov, "Nonlinear feedback design for fixed-time stabilization of linear control systems," IEEE Transactions on Automatic Control, vol. 57, no. 8, pp. 2106-2110, 2011.

[34] Z. Zuo, Q.-L. Han, B. Ning, X. Ge, and X.-M. Zhang, "An overview of recent advances in fixed-time cooperative control of multi-agent systems," IEEE Transactions on Industrial Informatics, vol. 14, no. 6, pp. 2322-2334, 2018.

[35] L. Wang, M.-F. Ge, Z. Zeng, and J. Hu, "Finite-time robust consensus of nonlinear disturbed multiagent systems via twolayer event-triggered control," Information Sciences, vol. 466, pp. 270-283, 2018.

[36] Y. Su, C. Zheng, and P. Mercorelli, "Robust approximate fixed-time tracking control for uncertain robot manipulators," Mechanical Systems and Signal Processing, vol. 135, Article ID 106379, 2020.

[37] H. Du, G. Wen, D. Wu, Y. Cheng, and J. Lü, "Distributed fixed-time consensus for nonlinear heterogeneous multiagent systems," Automatica, vol. 113, Article ID 108797, 2020.

[38] Z. Zuo, B. Tian, M. Defoort, and Z. Ding, "Fixed-time consensus tracking for multiagent systems with high-order integrator dynamics," IEEE Transactions on Automatic Control, vol. 63, no. 2, pp. 563-570, 2017.

[39] C. Wang, G. Wen, Z. Peng, and X. Zhang, "Integral slidingmode fixed-time consensus tracking for second-order nonlinear and time delay multi-agent systems," Journal of the Franklin Institute, vol. 356, no. 6, pp. 3692-3710, 2019.
[40] B. Ning, Q. L. Han, and Z. Zuo, "Practical fixed-time consensus for integrator-type multi-agent systems: a time base generator approach," Automatica, vol. 105, pp. 406-414, 2019.

[41] L. Wang, Z. Zeng, and M. F. Ge, "A disturbance rejection framework for finite-time and fixed-time stabilization of delayed memristive neural networks," IEEE Transactions on Systems, Man, and Cybernetics: Systems, vol. 51, no. 2, pp. 905-915, 2019.

[42] J. Ni, L. Liu, C. Liu, and J. Liu, "Fixed-time leader-following consensus for second-order multiagent systems with input delay," IEEE Transactions on Industrial Electronics, vol. 64, no. 11, pp. 8635-8646, 2017.

[43] V. Parra-Vega, "Second order sliding mode control for robot arms with time base generators for finite-time tracking," Dynamics and Control, vol. 11, no. 2, pp. 175-186, 2001.

[44] Y. Zhang, Y. Yang, Y. Zhao, and G. Wen, "Distributed finitetime tracking control for nonlinear multi-agent systems subject to external disturbances," International Journal of Control, vol. 86, no. 1, pp. 29-40, 2013.

[45] W. H. Zhu, "Comments on robust tracking control for rigid robotic manipulators," IEEE Transactions on Automatic Control, vol. 45, no. 8, pp. 1577-1580, 2000. 